\title{
Generalized Local Maximum Principles for Finite-Difference Operators
}

\author{
By Achi Brandt
}

\begin{abstract}
The generalized local maximum principle for a difference operator $L_{h}$ asserts that if $L_{h} u(x)>0$ then $\Gamma u$ cannot attain its positive maximum at the net-point $x$. Here $\Gamma$ is a local net-operator such that $\Gamma u=u+O(h)$ for any smooth function $u$. This principle, with simple forms of $\Gamma$, is proved for some quite general classes of second-order elliptic operators $L_{h}$, whose associated global matrices are not necessarily monotone. It is shown that these generalized principles can be used for easy derivation of global a priori estimates to the solutions of elliptic difference equations and to their difference-quotients. Some examples of parabolic difference equations are also treated.
\end{abstract}

1. Introduction. The maximum principle is a very useful analytical tool in the study of second-order elliptic and parabolic differential equations. A simple observation from differential calculus, this principle essentially states that if a differential operator $L$ is properly elliptic or parabolic, and if $L u(x)>0$, then the function $u$ cannot attain a positive local maximum at the point $x$. This is a local property of $L$ (valid even for operators with wildly variable coefficients) from which many global properties easily follow. Given for example an elliptic equation $L u(x)=f(x)$ in some domain $\Omega$, with the value of $u$ being given on the boundary of $\Omega$, one can construct various related functions $u^{\prime}$ of some independent variable $x^{\prime}$, with related elliptic operators $L^{\prime}$ in some regions $\Omega^{\prime}$, such that $L^{\prime} u^{\prime}\left(x^{\prime}\right)>0$ for all $x^{\prime} \in \Omega^{\prime}$, and such that $u^{\prime}<0$ on the boundary of $\Omega^{\prime}$. Then, by the maximum principle, $u^{\prime}$ must be negative throughout $\Omega^{\prime}$. This is a global result which, for $x^{\prime}, \Omega^{\prime}, L^{\prime}$ and $u^{\prime}$ suitably chosen, turns out to yield global estimates either for $u$ itself or for its derivatives and Hölder coefficients, up to some suitable order. (Cf. Brandt [8].) The uniqueness and stability (continuous dependence on data) of solutions can be instantly deduced from such a priori estimates. Also, a relatively simple proof of the existence of solutions can be based on the "continuity method" (cf., e.g., Courant and Hilbert [12]), where such a priori estimates are central, and on the "Perron's method", which is again built on the maximum principle. Thus the entire existence-uniqueness-stability theory can be developed from the maximum principle in a very elementary way, in which, for example, integral calculus can be discarded altogether. A clear advantage of this approach is the relative ease with which equations with variable, even discontinuous, coefficients are handled.

Even more significant is the role that can be played by the maximum principle in the theory of discrete (computable) approximations to elliptic and parabolic equations,

Received December 10, 1971.

AMS (MOS) subject classifications (1970). Primary 65M10, 65M15, 65N10, 65N15; Secondary 35J25, 35K 15, 35K20. 39A10.

Key words and phrases. Elliptic difference operators, parabolic difference operators, boundary value problems, maximum principles, monotone matrices, a priori estimates, estimates for differencequotients. 
because here various other analytical tools, such as fundamental solutions, oblique transformations of coordinates, etc., are either unavailable or very inconvenient.

Unfortunately, for finite-difference operators $L_{h}$ the maximum principle is not always as straightforward as for their continuous counterpart $L$. The simplest case here is that of "positive type" operators, i.e., operators of the form

$$
L_{h} u(x) \equiv \frac{1}{h^{2}} \sum_{\nu} b_{\nu}(x, h) u(x+\nu h),
$$

where $x=\left(x_{1}, \cdots, x_{n}\right), \nu=\left(\nu_{1}, \cdots, \nu_{n}\right), \nu_{i}$ are integers, $h>0$ is the "mesh-size", and where the coefficients satisfy

$$
\sum_{\nu} b_{\nu}(x, h) \geqq 0 \quad \text { and } \quad b_{\mu}(x, h) \leqq 0 \quad \text { for all } \mu \neq 0 .
$$

For such operators $L_{h}$ we have the following local maximum principle, which is completely analogous to the one mentioned above for $L$.

LOCAL MAXIMUM PRINCIPLE. If $L_{h} u(x)>0$, then $u(x)$ is either negative or less than $u\left(x^{\prime}\right)$, for some neighboring net-point $x^{\prime}=x+\nu h$.

Indeed, by (1.1)-(1.2), the inequality $L_{h} u(x)>0$ can be rewritten in the form

$$
u(x)<\sum_{\nu \neq 0} \omega_{\nu} u(x+\nu h), \quad \text { where } \omega_{\nu} \geqq 0 \text { and } \sum_{\nu \neq 0} \omega_{\nu} \leqq 1,
$$

showing $u(x)$ to be less than a subaverage of its values at neighboring points.

This discrete maximum principle can be used, like its continuous analog, to establish existence, uniqueness and stability of the finite-difference solutions. Also, by a method that goes back to Gerschgorin [15], the maximum principle can be employed to appraise the rate of convergence, i.e., the order of the $L_{\infty}$ distance between the discrete and the continuous solutions. (Cf. Forsythe and Wasow [14, p. 283].) This method has been refined and extensively used by Collatz [11], Laasonen [16], Bramble and Hubbard [3], [4], Bramble [1], [2], Bramble, Hubbard and Thomée [6], and many others. Moreover, from the maximum principle one can derive estimates to differencequotients of the discrete solution (cf. [7], [8], [9]), which may then be used to establish convergence of the finite-difference solutions, yielding an alternative existence proof for the differential problem.

In all that work, however, only positive-type operators were considered, with some possible relaxation of condition (1.2) at points $x$ adjacent to boundaries. (With such a relaxation, the problem is said to be "essentially of positive type".) It was shown by Motzkin and Wasow ([17]; cf. also [4]) that any second-order uniformly elliptic operator has a formal difference approximation which is of positive type. But these approximations are often inconvenient for actual computations, and many "natural" difference operators used in practice are not of positive type. For such nonpositivetype operators the above local maximum principle no longer holds, and the existenceuniqueness-stability-convergence theory requires a further justification.

Nonpositive-type operators were treated by Bramble and Hubbard [5]. Their approach was to show (by means of a certain matrix factorization which takes into account the special form of $L_{h}$ near the boundary, or special boundary data) that when the local maximum principle fails, a global maximum principle, of the following type, may still hold. 
Global Maximum Principle. If $L_{h} u(x)>0$ for all points $x$ of a certain discrete domain $\Omega_{h}$, then $u$ attains its maximum on the boundary of $\Omega_{h}$.

This property of $L_{h}$, usually referred to as monotonicity of the associated matrix, is proved in [5] for three cases:

(i) The nine-point-cross $O\left(h^{4}\right)$ finite-difference Laplace operator in a general region. This case was slightly generalized (inclusion of lower order terms) by Price [18].

(ii) A thirteen-point $O\left(h^{10}\right)$ Laplace operator in a rectangle.

(iii) The "most natural" nine-point $O\left(h^{2}\right)$ approximation to the elliptic operator $\partial^{2} / \partial x^{2}+\partial^{2} / \partial y^{2}+\partial^{2} / \partial x \partial y$, in a rectangle, with vanishing boundary values.

In each case it is shown that the global maximum principle entails the desired estimate for the rate of convergence.

This approach, formalized in [10], has some disadvantages. First, it is essentially limited to operators whose associated matrix (or some "interior" submatrix) is monotone. This monotonicity, or the global maximum principle, fails in many interesting cases, such as case (iii) above, when the boundary conditions do not vanish. Moreover, global maximum principles, unlike the local principles, cannot be used for estimating difference-quotients of solutions. The global principles are also much more laborious to derive.

The approach of the present article is therefore different; namely, to show that even nonpositive-type elliptic difference operators still satisfy the local maximum principle, in the following generalized sense.

Generalized Local Maximum Principle. If $L_{h} u(x)>0$, then $\Gamma u(x)$ is either negative or less than $\Gamma u\left(x^{\prime}\right)$, for some neighboring net-point $x^{\prime}$.

$\Gamma$ is a simple net-operator which, roughly speaking, tends to the identity operator as the mesh-size shrinks to zero. For instance, for certain nonpositive-type operators $L_{h}$, a suitable $\Gamma$ may have the form

$$
\Gamma u(x)=\max _{|\nu| \leqq 1} \frac{u(x)-\alpha u(x+\nu h)}{1-\alpha} \quad(0<\alpha=\alpha(x)<1),
$$

where $\nu=\left(\nu_{1}, \cdots, \nu_{n}\right)$ and $|\nu|=\left|\nu_{1}\right|+\cdots+\left|\nu_{n}\right|$. The exact value of $\alpha(x)$ depends on $L_{h}(x)$ and, for constant coefficients operators, $\alpha$ is usually a constant.

In the language of the associated global matrices, one can say that our approach is to add dependent variables to the problem so that the resulting extended problem can be represented by a positive-type matrix.

In Section 3, simple two-dimensional elliptic difference operators are studied, which are not necessarily monotone, but for which there still exist simple generalized local maximum principles, with $\Gamma$ of the form (1.4). These simple cases serve to illustrate the efficiency with which generalized principles can replace the ordinary maximum principles as a tool for deriving all kinds of global a priori estimates. We estimate the solutions as well as their difference-quotients, for both constant-coefficients and variable-coefficients difference operators. The method is extendable also to higher-dimensional operators. Case (iii) above, from [5], is a special case here.

In Section 4, the more general class of two-dimensional nine-point-box elliptic difference operators is considered. By a certain local decomposition of $L_{h}$ we establish a generalized local maximum principle which holds if and only if $L_{h}$ is elliptic. For constant-coefficients homogeneous operators, the decomposition has the form

$$
-h^{2} L_{h}=\Lambda^{*} \Lambda-\lambda^{2},
$$


where $\lambda$ is a positive scalar, and $\Lambda$ and $\Lambda^{*}$ are a certain net operator and its adjoint, respectively. $\Lambda$ is usually of positive type, in which case $\Gamma$ can be taken as

$$
\Gamma u(x)=\max \left\{u(x), \Lambda u(x) / \lambda, \Lambda^{*} u(x) / \lambda\right\} .
$$

If $\Lambda$ is not of positive type, then it can itself be decomposed, and the form of $\Gamma$ gets somewhat more complicated. (See Section 4.5.)

The problem of the $O\left(h^{4}\right)$ nine-point-cross difference approximation (case (i) above) is treated in Section 5. Using a generalized local maximum principle, we establish the global principle for that operator on a general domain. This is a slight strengthening of the results of Bramble and Hubbard [5] and Price [18], who established the global principle for mesh-lined regions only. Also, the derivation here is considerably simpler.

Finally, in Section 6 we consider several simple cases of parabolic difference operators. Again, generalized local maximum principles are demonstrated and then employed for some simple a priori estimates.

The remarkable feature of proofs via local maximum principles is their direct and easy applicability to equations with variable, even discontinuous, coefficients. Such a principle is usually an easy-to-derive local property that has extensive global implications. The main drawback, at present, seems to be the lack of generality. We treat in this paper fairly general classes of two-dimensional operators, but there is no general theory to tell us what the actual limits of the method are. One may conjecture, for example, that a generalized local maximum principle can be formulated for any difference operator which is second-order and elliptic in the sense of Thomée [19]. This, however, is not yet generally proved even for two-dimensional operators. It poses an interesting algebraic problem, which is probably manageable, because it deals only with the local form of $L_{h}$.

Thus, at this point, the method of generalized local maximum principles is not yet a general theory, but rather a collection of techniques, easily and successfully applied for many interesting classes of difference operators.

2. Finite-Difference Notation. Let $R$ be a bounded open domain in the $(x, y)$ plane, with boundary $B$, its closure denoted by $\bar{R}=R \cup B$. Let $h$ be a small positive constant-the "mesh size". In the usual manner, we impose a square net on the plane, that is, lines $x=i h$ and lines $y=j h$, where $i$ and $j$ are integers. The set $R_{h}$ will consist of all those line intersections that belong to $R$. The set of point-intersections of these lines with $B$ will be denoted $B_{h}$. We further define

$$
\begin{aligned}
\bar{R}_{h} & =R_{h} \cup B_{h}, \\
R_{h, k} & =R_{h} \cap\left\{(x, y):\left(x+\theta_{1} h, y+\theta_{2} h\right) \in \bar{R} \text { for all }\left|\theta_{i}\right| \leqq k\right\}, \\
B_{h, k} & =R_{h} / R_{h, k} .
\end{aligned}
$$

Clearly, $B_{h, 1}=B_{h}$ if $R$ is a "mesh-lined domain", i.e., if its boundary $B$ is composed only of segments of mesh lines.

The translation operator $T_{\alpha \beta}$ is defined for points $(x, y)$ in the plane and for functions $u(x, y)$ by the identities

$$
T_{\alpha \beta}(x, y)=(x+\alpha h, y+\beta h), \quad T_{\alpha \beta} u(x, y)=u(x+\alpha h, y+\beta h),
$$


from which it follows that

$$
T_{\alpha \beta} T_{\gamma \delta}=T_{\gamma \delta} T_{\alpha \beta}=T_{\alpha+\gamma, \beta+\delta} .
$$

The following notation will be used for finite-difference operators, with obvious analogy to the corresponding differential operators:

$$
\begin{aligned}
& \partial_{x}^{+}=\left(T_{10}-T_{00}\right) / h, \quad \partial_{x}^{-}=\left(T_{00}-T_{-10}\right) / h, \\
& \partial_{y}^{+}=\left(T_{01}-T_{00}\right) / h, \quad \partial_{y}^{-}=\left(T_{00}-T_{0-1}\right) / h, \\
& \partial_{x}=\frac{1}{2}\left(\partial_{x}^{+}+\partial_{x}^{-}\right)=\left(T_{10}-T_{-10}\right) /(2 h), \quad \partial_{y}=\frac{1}{2}\left(\partial_{y}^{+}+\partial_{y}^{-}\right)=\left(T_{01}-T_{0-1}\right) /(2 h), \\
& \partial_{x x}=\partial_{x}^{+} \partial_{x}^{-}=\left(T_{10}-2 T_{00}+T_{-10}\right) / h^{2}, \quad \partial_{y y}=\partial_{y}^{+} \partial_{y}^{-}=\left(T_{01}-2 T_{00}+T_{0-1}\right) / h^{2}, \\
& \partial_{x y}^{+}=\frac{1}{2}\left(\partial_{x}^{+} \partial_{y}^{+}+\partial_{x}^{-} \partial_{y}^{-}\right), \quad \partial_{x y}^{-}=\frac{1}{2}\left(\partial_{x}^{+} \partial_{y}^{-}+\partial_{x}^{-} \partial_{y}^{+}\right), \\
& \partial_{x y}=\frac{1}{2}\left(\partial_{x y}^{+}+\partial_{x y}^{-}\right)=\left(T_{11}-T_{1-1}-T_{-11}+T_{-1-1}\right) /\left(4 h^{2}\right), \\
& \Delta_{h}=\partial_{x x}+\partial_{y y} .
\end{aligned}
$$

We shall also use the following fourth-order approximations:

$$
\begin{aligned}
& \partial_{x}^{(4)}=\left(T_{-20}-8 T_{-10}+8 T_{10}-T_{20}\right) /(12 h), \\
& \partial_{y}^{(4)}=\left(T_{0-2}-8 T_{0-1}+8 T_{01}-T_{02}\right) /(12 h), \\
& \partial_{x x}^{(4)}=\left(-T_{-20}+16 T_{-10}-30 T_{00}+16 T_{10}-T_{20}\right) /\left(12 h^{2}\right), \\
& \partial_{y y}^{(4)}=\left(-T_{0-2}+16 T_{0-1}-30 T_{00}+16 T_{01}-T_{02}\right) /\left(12 h^{2}\right) .
\end{aligned}
$$

3. Simple Illustrations for Elliptic Equations. To illustrate the idea of generalized local maximum principles, and a variety of their applications, let us first consider the simple difference operator with constant coefficients

$$
L_{h} \equiv a \partial_{x x}+2 b \partial_{x y}+c \partial_{y y} \quad(a>0, c>0,|b| \leqq a c /(a+c)),
$$

which is, of course, consistent with a second-order elliptic differential operator. Introducing the auxiliary net-operators

$$
\begin{gathered}
\Lambda=\frac{T_{00}-\alpha T_{10}}{1-\alpha}, \quad \Lambda^{*}=\frac{T_{00}-\alpha T_{-10}}{1-\alpha}, \text { where } \alpha=\frac{|b|}{2 c}, \\
\Gamma \phi(P)=\max \left\{\phi(P), \Lambda \phi(P), \Lambda^{*} \phi(P)\right\},
\end{gathered}
$$

we can immediately state the following local principle:

Proposition 3.1. If $L_{h} u(P)>0$, then $\Gamma u$ does not attain its maximum at the point $P$.

Proof. Suppose, for definiteness, that $b \geqq 0$. Using the ad hoc notation

$$
\begin{array}{rlrl}
u_{i j} & =u\left(T_{i j} P\right), & \Lambda_{i j} & =\Lambda u\left(T_{i j} P\right), \\
\Lambda_{i j}^{*} & =\Lambda^{*} u\left(T_{i j} P\right), & \Gamma_{i j}=\Gamma u\left(T_{i j} P\right),
\end{array}
$$

we can rearrange the inequality $L_{h} u(P)>0$ into any one of the following forms:

$$
u_{00}<\frac{1}{2(a+c)}\left\{a\left(u_{10}+u_{-10}\right)+\frac{b}{2}\left(u_{11}+u_{-1-1}\right)+\frac{2 c-b}{2}\left(\Lambda_{01}^{*}+\Lambda_{0-1}\right)\right\},
$$




$$
\begin{aligned}
\Lambda_{00}< & \frac{1}{2(a+c)(1-\alpha)} \\
& \cdot\left\{(a-2 \alpha(a+c)) u_{10}+a u_{-10}+\frac{b}{2}\left(u_{11}+u_{-1-1}\right)+\frac{2 c-b}{2}\left(\Lambda_{01}^{*}+\Lambda_{0-1}\right)\right\}, \\
\Lambda_{00}^{*}< & \frac{1}{2(a+c)(1-\alpha)} \\
& \cdot\left\{(a-2 \alpha(a+c)) u_{-10}+a u_{10}+\frac{b}{2}\left(u_{11}+u_{-1-1}\right)+\frac{2 c-b}{2}\left(\Lambda_{01}^{*}+\Lambda_{0-1}\right)\right\} .
\end{aligned}
$$

In each of these inequalities, the right-hand side is seen to be a weighted average (i.e., the coefficients are positive and their sum is 1) of quantities like $u_{i j}, \Lambda_{i j}$ and $\Lambda_{i j}^{*}$, where each of these quantities is dominated by

$$
\bar{\Gamma}=\max \left\{\Gamma_{10}, \Gamma_{11}, \Gamma_{01}, \Gamma_{-10}, \Gamma_{-1-1}, \Gamma_{0-1}\right\} \text {. }
$$

Hence we have $u_{00}<\bar{\Gamma}, \Lambda_{00}<\bar{\Gamma}, \Lambda_{00}^{*}<\bar{\Gamma}$, and therefore also $\Gamma_{00}<\bar{\Gamma}$. Q.E.D.

In case $b<0$, we replace $u_{11}, u_{-1-1}, \Lambda_{01}^{*}$ and $\Lambda_{0-1}$ in the above inequalities by $u_{1-1}, u_{-11}, \Lambda_{01}$ and $\Lambda_{0-1}^{*}$, respectively.

Note that the requirement on $|b|$ in (3.1) is designed to ensure nonnegativity of the coefficient $a-2 \alpha(a+c)$ in the above inequalities.

Remark 3.1. If $\phi(P)$ is defined only for points $P$ of some restricted domain, and if $b \neq 0$, then $\Gamma \phi(P)$ is not well defined for boundary points $P$ where $\phi\left(T_{10} P\right)$ or $\phi\left(T_{-10} P\right)$ are not defined. On the other hand, the above proof shows that $\Lambda_{01}^{*}$ and $\Lambda_{0-1}$ should be introduced only when $L_{h} u(P)$ is well defined. We therefore modify (3.3) and will generally use the definition

$$
\begin{aligned}
\Gamma \phi(P) & =\phi(P), \quad \text { if either } \phi\left(T_{-10} P\right) \text { or } \phi\left(T_{10} P\right) \text { are undefined, } \\
& =\max \left\{\phi(P), \Lambda \phi(P), \Lambda^{*} \phi(P)\right\}, \quad \text { otherwise. }
\end{aligned}
$$

Proposition 3.1 and its proof remain valid, because $L_{h} u(P)$ is assumed to be defined, and hence, by $\left(3.3^{\prime}\right)$, we still have $\Lambda_{01}^{*} \leqq \Gamma_{01} \leqq \bar{\Gamma}$ and $\Lambda_{0-1} \leqq \Gamma_{0-1} \leqq \bar{\Gamma}$. Other modifications of (3.3) are also possible.

3.1. Global Estimates. Observe now that for $b=0$ we have $\Gamma \phi=\phi$, and the above proposition reproduces the classical maximum principle. For $b \neq 0$, however, $L_{h}$ is not of positive type and a classical local maximum principle does not exist. Nevertheless, the generalized-local principle of Proposition 3.1 can serve equally well in deriving global a priori estimates. As an easy example consider

Proposition 3.2. Let $R$ be the mesh-lined rectangle $R=\{(x, y):|x| \leqq \bar{x},|y| \leqq \bar{y}\}$, where $\bar{x} / h$ and $\bar{y} / h$ are integers. Assume $L_{h} u \geqq-\bar{l}$ in $R_{h}$ and $|u| \leqq \bar{u}$ on the boundary $B_{h}$, where $\bar{l} \geqq 0$ and $\bar{u}$ are constants. Then

$$
u(x, y) \leqq \frac{1}{2 c}\left(\bar{y}^{2}-y^{2}\right) \bar{l}+\frac{2 c+|b|}{2 c-|b|} \bar{u} \quad\left((x, y) \in R_{h}\right) .
$$

Proof. Writing $v=u-\psi$ where $\psi(x, y)=\left(\bar{y}^{2}-y^{2}\right) l / 2 c$, with $l>\bar{l}$, we clearly have $L_{h} v=L_{h} u+l>0$, and hence, by Proposition 3.1, $\Gamma v$ must attain its maximum at a point $\left(x_{0}, y_{0}\right) \in B_{h}$. In case $\left|x_{0}\right|=\bar{x}$, we have (cf. Remark 3.1) $\Gamma v\left(x_{0}, y_{0}\right)=$ $v\left(x_{0}, y_{0}\right) \leqq \bar{u}$. For $\left|x_{0}\right|<\bar{x}$ we must have $\left|y_{0}\right|=\bar{y}$ and hence, for some $\left|x_{1}-x_{0}\right| \leqq h$, 


$$
\Gamma v\left(x_{0}, y_{0}\right)=\frac{v\left(x_{0}, y_{0}\right)-\alpha v\left(x_{1}, y_{0}\right)}{1-\alpha}=\frac{u\left(x_{0}, y_{0}\right)-\alpha u\left(x_{1}, y_{0}\right)}{1-\alpha} \leqq \frac{1+\alpha}{1-\alpha} \bar{u} .
$$

Thus, in either case, $v(x, y) \leqq \Gamma v(x, y) \leqq \Gamma v\left(x_{0}, y_{0}\right) \leqq(1+\alpha) \bar{u} /(1-\alpha)$, which, upon writing $\alpha$ from (3.2) and letting $l \rightarrow \bar{l}$, yields (3.4). Q.E.D.

COROLLARY 3.1. If $L_{h} u \geqq 0$ in the rectangle $R_{h}$ and $u=0$ on the boundary $B_{h}$, then $u \leqq 0$ everywhere in $\bar{R}_{h}$.

This corollary, for $a=2 b=c=1$, is the maximum theorem proved by Bramble and Hubbard [5, Section VI]. Tracing through the last proof one can see that on the vertical boundaries $\{|x|=\bar{x},|y|<\bar{y}\}$, it is enough to assume in the corollary that $u \leqq 0$ (instead of $u=0$ ). By symmetry, the same conclusion holds in the case where $u$ is nonpositive on the horizontal boundaries $\{|x|<\bar{x},|y|=\bar{y}\}$ and vanishes on the rest of the boundary. In a third case, where $u$ vanishes on all boundaries except for the corners, the same conclusion is drawn provided $u(x, y) \leqq 0$ at the two corners where $b x y>0$, while $u(x, y) \geqq 0$ at the two other corners.* Regarding more general problems as superpositions of these three cases, we get the following strengthening of the corollary:

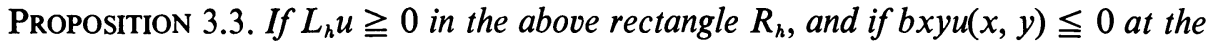
corners of $R$ and $u \leqq 0$ on the rest of its boundary, then $u \leqq 0$ throughout $R_{h}$.

The use of the generalized local maximum principle is in no way confined to rectangular regions. With a little more effort we can establish, for example, the more general

Propostition 3.4. Let $R$ be a general mesh-lined domain contained in the strip $\{(x, y):|y| \leqq \bar{y}\}$. Assume

$$
\begin{aligned}
\left|L_{h} u(P)\right| & \leqq \bar{l} & & \left(P \in R_{h}\right), \\
|u(Q)| & \leqq \bar{u} & & \left(Q \in B_{h}=B_{h, 1}\right) .
\end{aligned}
$$

Then

$$
|u(P)| \leqq \frac{b^{2}+4 c^{2}}{(2 c-|b|)^{2}} \bar{u}+\frac{\bar{y}^{2}}{2 c-|b|} \bar{l} \quad\left(P \in \bar{R}_{h}\right) .
$$

Proof. Take any $l>\bar{l}$. Introduce again the "comparison function" $\psi(x, y)=$ $l\left(\bar{y}^{2}-y^{2}\right) / 2 c$ and consider the difference $v=u-\psi$. By (3.5) we have $L_{h} v(P)>0$ for all $P \in R_{h}$, and hence, by Proposition 3.1, $\Gamma v$ must attain its maximum at a point $Q_{1} \in B_{h}$; that is, for every $P \in \bar{R}_{h}$ we have

$$
\begin{aligned}
\Gamma u(P)-\psi(P) & =\Gamma v(P) \leqq \Gamma v\left(Q_{1}\right) \leqq \Gamma u\left(Q_{1}\right) \\
& =\frac{u(Q)-\alpha u\left(Q_{1}^{\prime}\right)}{1-\alpha} \leqq \frac{\bar{u}}{1-\alpha}+\frac{\alpha}{1-\alpha}\left|u\left(Q_{1}^{\prime}\right)\right|,
\end{aligned}
$$

where $Q_{1}^{\prime} \in B_{h, 2}$. Similarly, replacing $u$ by $-u$, we get, for some $Q_{2}^{\prime} \in B_{h, 2}$, putting

* To see this, e.g., for $b>0$, one has to slightly modify the definition $\left(3.3^{\prime}\right)$ at some points,

$$
\begin{array}{ll}
\Gamma u(P)=\max \left(u(P), \Lambda^{*} u(P)\right) & \text { for } P=( \pm(\bar{x}-h), \bar{y}), \\
\Gamma u(P)=\max (u(P), \Lambda u(P)) & \text { for } P=( \pm(\bar{x}-h),-\bar{y}),
\end{array}
$$

and, for convenience, $\Gamma u( \pm(x,-y))=0$. Observe then that the proof of Proposition 3.1 still applies for all $P \in R_{h}$, while $\Gamma u(Q) \leqq 0$ for all $Q \in B_{h}$. 


$$
\Gamma(-u(P))-\psi(P) \leqq \frac{\bar{u}}{1-\alpha}+\frac{\alpha}{1-\alpha}\left|u\left(Q_{2}^{\prime}\right)\right| \quad\left(P \in \bar{R}_{h}\right),
$$

which, together with the previous inequality, gives

$$
|\Gamma| u(P) \leqq \frac{1}{1-\alpha} \bar{u}+\frac{\alpha}{1-\alpha}\left|u\left(Q^{\prime}\right)\right|+\psi(P),
$$

where $Q^{\prime}$ is either $Q_{1}^{\prime}$ or $Q_{2}^{\prime}$ and $|\Gamma| u=\max \{\Gamma u, \Gamma(-u)\}=\max \left\{|u|,|\Lambda u|,\left|\Lambda^{*} u\right|\right\}$. From this definition of $|\Gamma|$ it follows that $|\Gamma| u\left(Q^{\prime}\right) \geqq\left|u\left(Q^{\prime}\right)-\alpha u(Q)\right| /(1-\alpha)$ for some $Q \in B_{h}$, and hence, by (3.6),

$$
\frac{1}{1-\alpha}\left|u\left(Q^{\prime}\right)\right| \leqq|\Gamma| u\left(Q^{\prime}\right)+\frac{\alpha}{1-\alpha} \bar{u} .
$$

This, together with (3.8), gives

$$
|\Gamma| u(P) \leqq \frac{1+\alpha^{2}}{1-\alpha} \bar{u}+\alpha|\Gamma| u\left(Q^{\prime}\right)+\psi(P) \quad\left(P \in R_{h}\right) .
$$

Using this inequality for $P=Q^{\prime}$ we get an estimate for $|\Gamma| u\left(Q^{\prime}\right)$ which, when plugged back into (3.10), completes the proof.

3.2. Estimates of Difference-Quotients. Local maximum principles can be used, in a direct and elementary way, not only for estimating the magnitude of elliptic equations solutions, but also for estimating their difference-quotients (or derivatives, in the continuous case). Cf. Brandt [7], [8], [9]. We shall show here, again through simple examples, that the same can be accomplished with generalized local maximum principles. The first example here corresponds to the one in the introduction to [8]. As in that paper, the method can be vastly extended to produce various types of interior estimates, such as Schauder estimates, Hölder continuity of solutions to equations with discontinuous coefficients, etc. The second example here will be of a uniform (up-to-the-boundary) estimate.

Proposition 3.5. Let $R$ be a general domain whose boundary has distance $d+2 h$ from $O$ (the origin). Let $L_{h}$ now be defined by

$$
L_{h}=a \partial_{x x}+2 b \partial_{x y}+(c+\gamma) \partial_{y y} \quad(a>0, c>0, \gamma>0,|b|<a c /(a+c+\gamma)) \text {, }
$$

where $a, b, c, \gamma$ are constant. Assume

Then

$$
\begin{aligned}
\left|L_{h} u(P)\right| & \leqq \bar{l} & & \left(P \in R_{h}\right), \\
|u(P)| & \leqq \bar{u} & & \left(P \in \bar{R}_{h}\right) .
\end{aligned}
$$

Proof. Our approach is to view the difference

$$
\hat{u}(x, y, z)=\frac{1}{2}[u(x, y+z)-u(x, y-z)]
$$

as a function of the three variables $(x, y, z)$, defined on the domain

$$
\hat{R}_{h}=\left\{(x, y, z) \mid \frac{x}{h}, \frac{y}{h}, \frac{z}{h} \text { are integers; }|x|,|y|<\frac{d}{3} ; 0<z \leqq \frac{d}{3}\right\} .
$$


Introducing the three-variable discrete elliptic operator

$$
\hat{L}_{h}=a \partial_{x x}+2 b \partial_{x y}+c \partial_{y y}+\gamma \partial_{z z},
$$

we observe that

$$
\left|\hat{L}_{h} \hat{u}(x, y, z)\right|=\frac{1}{2}\left|L_{h} u(x, y+z)-L_{h} u(x, y-z)\right| \leqq \bar{l}, \quad(x, y, z) \in \hat{R}_{h} .
$$

Hence, using any "comparison function" of the form

$$
\psi(x, y, z)=\left(A \frac{a+c+\gamma}{\gamma}+\frac{l}{2 \gamma}\right)\left(\frac{d}{3} z-z^{2}\right)+A\left(x^{2}+y^{2}+z^{2}\right) \quad(l>\bar{l}),
$$

it follows that

$$
\hat{L}_{h}(\hat{u}-\psi)=\hat{L}_{h} \hat{u}+l>0 .
$$

To this inequality we can apply a local maximum principle in the same way as in Proposition 3.1. Indeed, if we define

$$
\begin{aligned}
& \Lambda \phi(x, y, z)=\frac{\phi(x, y, z)-\alpha \phi(x+h, y, z)}{-1-\alpha}, \\
& \Lambda^{*} \phi(x, y, z)=\frac{\phi(x, y, z)-\alpha \phi(x-h, y, z)}{1-\alpha}, \\
& \Gamma \phi(P)=\max \left\{\phi(P), \Lambda \phi(P), \Lambda^{*} \phi(P)\right\}, \quad \text { if this is well defined, } \\
& =\phi(P), \\
& \text { otherwise, }
\end{aligned}
$$

where $\alpha=|b| / 2 c$, and if, for example, $b \geqq 0$, then the inequality $\hat{L}_{h} \phi(x, y, z)>0$ can be rearranged into the form

$$
\begin{aligned}
& \frac{(2 c-b)(a+c+\gamma)}{c} \Lambda \phi(x, y, z) \\
& <a \phi(x-h, y, z)+\frac{2 c-b}{2}\left[\Lambda^{*} \phi(x, y+h, z)+\Lambda \phi(x, y-h, z)\right] \\
& \quad+\frac{b}{2}[\phi(x+h, y+h, z)+\phi(x-h, y-h, z)] \\
& \quad+\left[a-\frac{b}{c}(a+c+\gamma)\right] \phi(x+h, y, z)+\gamma[\phi(x, y, z+h)+\phi(x, y, z-h)]
\end{aligned}
$$

which shows $\Lambda \phi(x, y, z)$ to be less than a weighted average of $\phi, \Lambda \phi$ and $\Lambda^{*} \phi$ at neighboring points. Two similar rearrangements show $\Lambda^{*} \phi(x, y, z)$ and $\phi(x, y, z)$ also to be less than weighted averages of $\phi, \Lambda \phi$ and $\Lambda^{*} \phi$ at neighboring points and hence $\Gamma \phi$ cannot attain its maximum at $(x, y, z)$. In particular, taking $\phi=\hat{u}-\psi$, we see from (3.12) that $\Gamma \phi$ must attain its maximum on the boundary of $\hat{R}_{h}$. But on that boundary $\Gamma \phi$ is seen to be nonpositive, once the constant $A$ is chosen large enough, e.g.,

$$
A=\frac{9}{d^{2}} \frac{1+\alpha}{1-\alpha} \bar{u} .
$$

Thus $\Gamma \phi$ must be nonpositive throughout $\hat{R}_{h}$, which implies $u \leqq \psi$. Similarly, one can 
show $-\hat{u} \leqq \psi$, and hence $|\hat{u}| \leqq \psi$ throughout $\hat{R}_{h}$, or

$\frac{1}{2}|u(x, y+z)-u(x, y-z)|$

$$
\leqq \frac{l}{2 \gamma}\left(\frac{d}{3} z-z^{2}\right)+\frac{9}{d^{2}} \frac{1+\alpha}{1-\alpha} \bar{u}\left[x^{2}+y^{2}+z^{2}+\frac{a+c+\gamma}{\gamma}\left(\frac{d}{3} z-z^{2}\right)\right] \text {. }
$$

For $x=y=0, z=h$ and $l \rightarrow \bar{l}$, this last inequality yields (3.11). Q.E.D.

The estimate (3.11) is an interior estimate; it breaks down when the distance to the boundary $(d)$ shrinks to zero. To obtain uniform estimates, through the use of the maximum principle, one should proceed in two steps.

The first step is to obtain "from the boundary" estimates. By this we mean estimates of differences (or difference-quotients) with "legs" on the boundary; for example, an estimate of

$$
\sup _{\sigma \in B_{h}} \sup _{P \in R_{h}}|u(P)-u(Q)| /|P-Q|,
$$

where $|P-Q|$ designates the distance between $P$ and $Q$. Such an estimate can be easily obtained as a usual application of the maximum principle, by choosing, for each boundary point $Q$, a comparison function $(\psi)$ that coincides with $u$ at $Q$. For instance, if in Proposition 3.2 the boundary values vanish $(\bar{u}=0)$, then (3.4) can be written as

$$
\frac{u(x, y)-u\left(x^{\prime}, \bar{y}\right)}{\bar{y}-y} \leqq \frac{1}{c} \bar{y} \bar{l} \quad\left(|x| \leqq \bar{x},\left|x^{\prime}\right| \leqq \bar{x}\right),
$$

which is actually a "from-the-boundary" estimate.

From that type of estimates the uniform estimates are then obtained. The technique at this second step is generally the same as with interior estimates; namely, the maximum principle is applied to a suitable elliptic difference operator $\left(\hat{L}_{h}\right)$ on a higherdimensional domain $\left(\hat{R}_{h}\right)$. For example, we shall prove

Proposition 3.6. Let $R$ be any mesh-lined domain such that

$$
\sup \left\{y_{1}-y_{2}:\left(x, y_{1}\right) \in R,\left(x, y_{2}\right) \in R\right\} \leqq d,
$$

and let $L_{h}$ be defined as in Proposition 3.5. Assume

$$
\left|L_{h} u(P)\right| \leqq \bar{l} \quad\left(P \in R_{h}\right),
$$

and

$$
|u(P)-u(Q)| /|P-Q| \leqq \bar{m} \quad\left(P \in \bar{R}_{h}, Q \in B_{h}\right)
$$

Then

$$
\left|\partial_{y} u(P)\right| \leqq \frac{2 c+2|b|}{2 c-|b|} \bar{m}+\frac{d}{4 \gamma} \bar{l} \quad\left(P \in R_{h}\right) .
$$

Proof. $\hat{u}, \hat{L}_{h}, \alpha, \Lambda, \Lambda^{*}$ and $\Gamma$ are defined as in the previous proof, so that again we have $\left|\hat{L}_{h} \hat{u}\right| \leqq \bar{l}$ and the same local maximum principle, namely, that, for an arbitrary function $\phi, \Gamma \phi$ cannot attain its maximum at a point where $\hat{L}_{h} \phi$ is positive. As our higher-dimensional domain we take here

$$
\hat{R}_{h}=\left\{(x, y, z): \frac{x}{h}, \frac{y}{h}, \frac{z}{h} \text { are integers, } z>0,(x, y+z) \in R_{h},(x, y-z) \in R_{h}\right\} .
$$


For comparison function, we select

$$
\psi(x, y, z)=\frac{1+2 \alpha}{1-\alpha} \bar{m} z+\frac{l}{2 \gamma}\left(\frac{d}{2} z-z^{2}\right),
$$

which is easily seen to satisfy $\hat{L}_{h}(\hat{u}-\psi)>0$ in $\hat{R}_{h}$ and $\Gamma(\hat{u}-\psi) \leqq 0$ on its boundary $\partial \hat{R}_{h}$. (Indeed, if $(x, y, z) \in \partial R_{h}$, then either $z=0$ and hence $\Gamma \hat{u}=\psi=0$, or $(x, y+z)$ or $(x, y-z) \in B_{h}$, in which case $\Gamma \hat{u}(x, y, z)$ can be estimated by (3.14) and $\Gamma \hat{u}(x, y, z)$ $\leqq \psi(x, y, z)$ will easily follow.) Thus, by the maximum principle, $\Gamma(\hat{u}-\psi) \leqq 0$ throughout $\hat{R}_{h}$, implying $\hat{u} \leqq \psi$. Similarly, $-\hat{u} \leqq \psi$ and hence $|\hat{u}| \leqq \psi$, which involves (3.15). Q.E.D.

Combining Propositions 3.6 and 3.2 (cf. (3.13a)) one can get

CoROLlary 3.2. Let $R$ be a mesh-lined rectangle as in Proposition 3.2 and let $L_{h}$ be defined as in Proposition 3.5. If $\left|L_{h} u\right| \leqq \bar{l}$ in $R_{h}$ and $u$ vanishes on $B_{h}$ then

$$
\left|\partial_{y} u(P)\right| \leqq \frac{2 c+2|b|}{2 c-|b|} \bar{l} \max \left\{\frac{\bar{y}}{c+\gamma}, \frac{\bar{x}}{a}\right\}+\frac{\bar{y} \bar{l}}{2 \gamma} \quad\left(P \in R_{h}\right) .
$$

3.3. Variable Coefficients. One of the main advantages of the maximum principle as an analytical tool is that it is a simple local property from which global properties can be directly derived, with essentially no extra complications for the case of nonconstant coefficients.

Suppose for instance that $a, b$ and $c$ in (3.1) are nonconstant functions. Then $\alpha=|b| /(2 c)$ is also a variable function. We can still introduce $\Gamma$ as (3.3), but with (3.2) taking here the form

$$
\begin{aligned}
\Lambda \phi(x, y) & =\max _{\alpha=\alpha(x, y \pm h)} \frac{\phi(x, y)-\alpha \phi(x+h, y)}{1-\alpha}, \\
\Lambda^{*} \phi(x, y) & =\max _{\alpha=\alpha(x, y \pm h)} \frac{\phi(x, y)-\alpha \phi(x-h, y)}{1-\alpha} .
\end{aligned}
$$

(Each max is of course extended only over those values of $\alpha=\alpha(x, y \pm h)$ which are well defined.) It is then easily observed that Proposition 3.1, together with its proof, remains valid, provided only that the previous requirement $|b|<a c /(a+c)$ is rewritten here as

$$
\max \left\{\frac{|b(x, y+h)|}{c(x, y+h)}, \frac{|b(x, y-h)|}{c(x, y-h)}\right\}<\frac{a(x, y)}{a(x, y)+c(x, y)} .
$$

(The only point to note in the proof is that $a, b, c$ now stand for $a(x, y), b(x, y)$ and $c(x, y)$, respectively, while $\alpha$ should be interpreted as $\alpha(x, y+h)$ in the inequality for $\Lambda_{00}$ and as $\alpha(x, y-h)$ in the inequality for $\Lambda_{00}^{*}$.)

With similar straightforward modifications (e.g., $\min c(x, y)$ replacing $c$ in the expressions for the comparison functions $\psi$ ), the global estimates, such as Propositions 3.2, 3.3 and 3.4, together with their proofs, can also be extended to the variablecoefficients case. Their new forms will be as follows:

PROPOSITION 3.2'. Let $R$ be the mesh-lined rectangle $R=\{(x, y):|x| \leqq \bar{x},|y| \leqq \bar{y}\}$, where $\bar{x} / h, \bar{y} / h$ are integers. Assume $a \partial_{x x} u+2 b \partial_{x y} u+c \partial_{y y} u \geqq-\bar{l}$ in $R_{h}$, where $a>0, c>0$ and (3.16) is satisfied in $R_{h}$. Assume further that $|u| \leqq \bar{u}$ on the boundary $B_{h}$. Then 


$$
u(x, y) \leqq \frac{1}{2}\left[\min _{|\xi|<\bar{x} ;|\eta|<\bar{j}} c(\xi, \eta)\right]^{-1}\left(\bar{y}^{2}-y^{2}\right) \bar{l}+\bar{u} \max _{|\xi|<\bar{x} ;|\eta|=\bar{y}-h} \frac{2 c(\xi, \eta)+b(\xi, \eta)}{2 c(\xi, \eta)-b(\xi, \eta)}
$$

Proposition 3.3'. Assume, in addition to (3.16), that $a>0$ and

$$
\max \left\{\frac{|b(x+h, y)|}{a(x+h, y)}, \frac{|b(x-h, y)|}{a(x-h, y)}\right\}<\frac{c(x, y)}{a(x, y)+c(x, y)} .
$$

If $a \partial_{x x} u+2 b \partial_{x y} u+c \partial_{y y} u \geqq 0$ in the above rectangle $R_{h}$, and if

$$
\begin{aligned}
b\left(x-\frac{x}{|x|} h, y-\frac{y}{|y|} h\right) x y u(x, y) & \leqq 0, \text { for }|x|=\bar{x},|y|=\bar{y}, \\
u(x, y) & \leqq 0, \text { otherwise on } B_{h},
\end{aligned}
$$

then $u \leqq 0$ throughout $R_{h}$.

Proposition 3.4'. Let $R$ be a general mesh-lined domain contained by the strip $\{(x, y):|y| \leqq \bar{y}\}$. Assuming (3.16) and $a>0$, if

$$
\begin{aligned}
\left|a(P) \partial_{x x} u(P)+2 b(P) \partial_{x y} u(P)+c(P) \partial_{y y} u(P)\right| \leqq \bar{l} & \left(P \in R_{h}\right), \\
|u(Q)| \leqq \bar{u} & \left(Q \in B_{h}=B_{h, 1}\right),
\end{aligned}
$$

then, for all $P \in R_{h}$, we have

$$
|u(P)| \leqq \frac{4 c c^{\prime}+\left|b b^{\prime}\right|}{4 c c^{\prime}-4|b| c^{\prime}+\left|b b^{\prime}\right|} \bar{u}+\frac{2 c c^{\prime}-c^{\prime} b}{4 c c^{\prime}-4|b| c^{\prime}+\left|b b^{\prime}\right|} \frac{\bar{y}^{2}}{\min c} \bar{l}
$$

where $b=b\left(x_{1}, y_{1}\right), c=c\left(x_{1}, y_{1}\right), b^{\prime}=b\left(x_{2}, y_{2}\right), c^{\prime}=c\left(x_{2}, y_{2}\right)$, and $\left(x_{1}, y_{1}\right)$ and $\left(x_{2}, y_{2}\right)$ are some two points in $B_{h, 3}$ such that $\left|x_{1}-x_{2}\right| \leqq h,\left|y_{1}-y_{2}\right| \leqq 2 h$.

In estimating difference-quotients, the modifications from constant to variable coefficients are less straightforward. For example, in estimating first-order differencequotients, the auxiliary elliptic operator $\hat{L}_{h}$ should be taken as 4-dimensional, acting on the differences $\hat{u}(x, y, \xi, \eta)=\frac{1}{2}[u(x+\xi, y+\eta)-u(x-\xi, y-\eta)]$, and should contain mixed terms like $\partial_{x \xi}, \partial_{x \eta}, \partial_{y \xi}, \partial_{y \eta}$. Such terms can take care of the variability in the coefficients from $(x+\xi, y+\eta)$ to $(x-\xi, y-\eta)$. Generally, proofs for interior estimates should be modelled after the methods presented in Brandt [8]. For uniform estimates, one should combine those methods with "from-the-boundary" estimates, such as (3.17), $\bar{u}=0$.

3.4. More General Operators. There is no difficulty in using the methods of this section in more general situations. For instance, it is easy to observe that adding to $L_{h}$ lower-order terms $\left(r \partial_{x}+s \partial_{y}+q\right)$ will not significantly change any of the above proofs (with the same $\Gamma$ 's), provided $r h$, $s h$ and $q h^{2}$ are so small that the coefficients of the weighted averages remain nonnegative. Also, higher-dimensional $L_{h}$ can be treated in a similar fashion, provided that its nonpositive-type terms (such as $b \partial_{x y}$ ) are suitably bounded. Thus, under restrictions similar to, but generally different from (3.16), generalized maximum principles can be introduced to wide classes of elliptic difference operators.

The trouble is, however, that (3.16) is still too restrictive. It can be relaxed; for example, in the constant-coefficients case, it may be replaced by the weaker condition

$$
b^{2}<a^{2} c /(a+c)
$$


One can check this upon taking $\alpha=(\sigma-c) /|b|$, where $\sigma=\left(b^{2}+c^{2}\right)^{1 / 2}$, and observing that Proposition 3.1 remains valid. The only changes in its proof are in the three rearrangements of the inequality $L_{h} u(P)>0$, which will now be the following (for $b>0)$ :

$$
\begin{aligned}
u_{00} & <\frac{1}{2(a+c)}\left\{a\left(u_{10}+u_{-10}\right)+\bar{\Lambda}\right\}, \\
\Lambda_{00} & <\frac{1}{(2 a+2 c-\alpha a)(1-\alpha)}\left\{a(1-\alpha) \Lambda_{-10}+[a-\alpha(2 a+2 c-\alpha a)] u_{10}+\bar{\Lambda}\right\}, \\
\Lambda_{00}^{*} & <\frac{1}{(2 a+2 c-\alpha a)(1-\alpha)}\left\{a(1-\alpha) \Lambda_{10}^{*}+[a-\alpha(2 a+2 c-\alpha a)] u_{-10}+\bar{\Lambda}\right\},
\end{aligned}
$$

where

$$
\bar{\Lambda}=\frac{b-\sigma+c}{2}\left(\Lambda_{11}^{*}+\Lambda_{-1-1}\right)+\frac{c b}{b+\sigma-c}\left(\Lambda_{01}^{*}+\Lambda_{0-1}\right) .
$$

But (3.18) is again too restrictive, because it is stronger than the ellipticity condition $b^{2}<a c$. General elliptic operators will be considered in the next section, by using more complex decompositions of $L_{h}$.

4. General Nine-Point ("Box") Elliptic Difference Equations. In this section, maximum principles will be described for general nine-point ("box") difference approximations to second-order elliptic operators,

$$
\begin{aligned}
L=a \frac{\partial^{2}}{\partial x^{2}}+2 b \frac{\partial^{2}}{\partial x \partial y}+c \frac{\partial^{2}}{\partial y^{2}}+r \frac{\partial}{\partial x}+s \frac{\partial}{\partial y}-q & \\
& \left(a>0, b^{2}<a c, q \geqq 0\right) .
\end{aligned}
$$

4.1. Decomposition, Ellipticity and Local Maximum Principle. We start with general nine-point homogeneous operators with constant coefficients, namely

$$
L_{h}=a \partial_{x x}+b^{+} \partial_{x y}^{+}+b^{-} \partial_{x y}^{-}+c \partial_{y y} \quad\left(b^{+}+b^{-}=2 b, b^{+} b^{-} \geqq 0\right),
$$

where $a, b^{+}, b^{-}, c$ are real constants. More explicitly,

$$
\begin{aligned}
L_{h}=\frac{1}{h^{2}}\left\{A \left(T_{10}+\right.\right. & \left.T_{-10}\right)+C\left(T_{01}+T_{0-1}\right)+\frac{b^{+}}{2}\left(T_{11}+T_{-1-1}\right) \\
& \left.-\frac{b^{-}}{2}\left(T_{1-1}+T_{-11}\right)-\left(2 A+2 C+b^{+}-b^{-}\right) T_{00}\right\},
\end{aligned}
$$

where $A=a+b^{-} / 2-b^{+} / 2, C=c+b^{-} / 2-b^{+} / 2$. The "classical" maximum principle (cf., e.g., Forsythe and Wasow [14]) holds for this difference operator if (and only if) it is of positive type; that is, if in (4.1b) the coefficients of all $T_{i j}$, except for $T_{00}$, are nonnegative. In all other cases, we would like to construct generalized maximum principles. For this end the following decomposition theorem will be instrumental.

Proposition 4.1. Assume that, in the above $L_{h}, 0<a c, 0<|b| \leqq \max \{a, c\}$. If (and only if) $L_{h}$ is not of positive type, then it can be uniquely decomposed as follows:

$$
h^{2} L_{h}=\lambda^{2} T_{00}-\Lambda^{*} \Lambda,
$$


where

$$
\begin{aligned}
\Lambda & =\lambda_{0} T_{00}-\lambda_{1} T_{\beta 0}-\lambda_{2} T_{\beta 1}-\lambda_{3} T_{01}, \\
\Lambda^{*} & =\lambda_{0} T_{00}-\lambda_{1} T_{-\beta 0}-\lambda_{2} T_{-\beta-1}-\lambda_{3} T_{0-1}, \\
\lambda & =\lambda_{0}-\lambda_{1}-\lambda_{2}-\lambda_{3}, \quad \beta=b /|b|,
\end{aligned}
$$

and where $\lambda_{0}, \lambda_{1}, \lambda_{2}, \lambda_{3}$ are nonnegative real numbers.

Proof. Take for example $b>0$, and hence $b^{+} \geqq 0, b^{-} \geqq 0, \beta=1$. Using (2.1), the requirement (4.2) is easily found to be equivalent to the following four nonlinear equations

$$
\begin{aligned}
\lambda_{0} \lambda_{2} & =\frac{1}{2} b^{+}, \quad \lambda_{1} \lambda_{3}=\frac{1}{2} b^{-}, \\
\lambda_{9} \lambda_{1}-\lambda_{2} \lambda_{3} & =A, \quad \lambda_{0} \lambda_{3}-\lambda_{2} \lambda_{1}=C .
\end{aligned}
$$

Denote $\lambda_{i j}=\lambda_{i} \lambda_{j}$. We get the pair of equations $\lambda_{01}-\lambda_{23}=A, \lambda_{01} \lambda_{23}=b^{+} b^{-} / 4$. Since $b^{+} b^{-} \geqq 0$, the only nonnegative solution to this pair of equations is

$$
\lambda_{01}=\frac{1}{2}\left[\left(A^{2}+b^{+} b^{-}\right)^{1 / 2}+A\right], \quad \lambda_{23}=\frac{1}{2}\left[\left(A^{2}+b^{+} b^{-}\right)^{1 / 2}-A\right] .
$$

Similarly

$$
\lambda_{03}=\frac{1}{2}\left[\left(C^{2}+b^{+} b^{-}\right)^{1 / 2}+C\right], \quad \lambda_{21}=\frac{1}{2}\left[\left(C^{2}+b^{+} b^{-}\right)^{1 / 2}-C\right] .
$$

If $b^{+} b^{-}>0$ then all $\lambda_{i j}>0$, and the $\lambda_{i}$ themselves are determined by

$$
\lambda_{i}=\left(\lambda_{i j} \lambda_{i k} / \lambda_{j k}\right)^{1 / 2} \text {. }
$$

If $b^{+}=0$ then $b^{-}=2 b>0$ and $C=c+b>0$, for which case (4.3) has a unique nonnegative solution $\left(\lambda_{2}=0\right)$ ). If $b^{-}=0$ then $b^{+}=2 b>0$ and $A C<0$ (otherwise either $L_{h}$ is of positive type or $A<0$ and $C<0$, which contradicts the condition $b \leqq \max \{a, c\}$; if $A<0$, it follows from (4.3) that $\lambda_{1}=0, \lambda_{23}=-A, \lambda_{03}=C$, $\lambda_{02}=b^{+} / 2$ and $\lambda_{0}, \lambda_{2}, \lambda_{3}$ are determined by (4.4c); if $C<0$, it follows from (4.3) that $\lambda_{3}=0, \lambda_{01}=A, \lambda_{21}=-C, \lambda_{02}=b^{+} / 2$ and $\lambda_{0}, \lambda_{1}, \lambda_{2}$ are determined by (4.4c). Q.E.D. (The "only if" part of the proposition is straightforward.)

Note that the ellipticity condition $b^{2}<a c$ nowhere really entered in the above proposition or proof. Its role will become apparent in the next proposition, which, in fact, will give a genuine finite-difference interpretation to the ellipticity of $L_{h}$.

Proposition 4.2. Let $L_{h}$, defined by (4.1) with $a>0$, be not of positive type, and let it be decomposed by (4.2). Then $\lambda>0$ if and only if $b^{2}<a c$.

Proof. From (4.3) we get

$$
\begin{aligned}
& a=\left(\lambda_{0}-\lambda_{3}\right)\left(\lambda_{2}+\lambda_{1}\right), \\
& b=\lambda_{0} \lambda_{2}+\lambda_{1} \lambda_{3}, \\
& c=\left(\lambda_{0}-\lambda_{1}\right)\left(\lambda_{2}+\lambda_{3}\right),
\end{aligned}
$$

from which it is easily verified that

$$
a c-b^{2}=\lambda\left(\lambda_{0} \lambda_{1} \lambda_{2}+\lambda_{0} \lambda_{1} \lambda_{3}+\lambda_{0} \lambda_{2} \lambda_{3}-\lambda_{1} \lambda_{2} \lambda_{3}\right)=\lambda \lambda_{*}, \text { say. }
$$

By (4.4d), $a>0$ implies $\lambda_{0}>\lambda_{3}$, hence $\lambda_{0} \lambda_{1} \lambda_{2} \geqq \lambda_{1} \lambda_{2} \lambda_{3}$, and therefore $\lambda_{*} \geqq 0$. For $\lambda_{*}=0$, we must have either $\lambda_{1}=\lambda_{3}=0$ or $\lambda_{2}=\lambda_{3}=0$ or $\lambda_{1}=\lambda_{2}=0$, all 
cases implying $L_{h}$ of positive type (with $a c=b^{2}$ and with arbitrary value of $\lambda$ ), in contradiction to the assumption. Therefore $\lambda_{*}>0$. Q.E.D.

For $\lambda>0$, the operator $\Lambda$ is of positive type. We thus have the interesting result that ellipticity of $L_{h}$ is equivalent to its either being of positive type or having positivetype decomposition. This "generalized positive-type" property leads directly to the following generalized local maximum principle.

Proposition 4.3. Let $L_{h}$, defined by (4.1), be elliptic, and let $\Gamma u$ be defined by

$$
\begin{aligned}
\Gamma u(Q) & =u(Q), & & \text { if } L_{h} \text { is of positive type, } \\
& =\max \{u(Q), \Lambda u(Q) / \lambda\}, & & \text { otherwise. }
\end{aligned}
$$

If $L_{h} u(P)>0$, then $\Gamma u$ cannot attain its maximum at $P$.

Remark 4.1. The above definition of $\Gamma u$ is a natural one, in the sense that the transition from one case to the other is continuous; i.e., when $L_{h}$ tends to a positivetype operator, $\Lambda u / \lambda$ tends to $u$. Indeed, it is straightforward from (4.3) that, for $L_{h}$ tending to an elliptic positive-type operator $\left(A \geqq 0, C \geqq 0, b^{+} \geqq 0, b^{-}=0,\left(\frac{1}{2} b^{+}\right)^{2}=\right.$ $\left.b^{2}<a c\right)$, we must have $\lambda_{0} \rightarrow+\infty$ and $\lambda_{i} \rightarrow 0(i=1,2,3)$.

Proof. If $L_{h}$ is of positive type, then $b^{+} \geqq 0, b^{-} \leqq 0, A \geqq 0, C \geqq 0$. Rewrite $L_{h} u(P)>0$ in the form

$$
\begin{gathered}
\left(2 A+2 C+b^{+}-b^{-}\right) u_{00}<A u_{10}+A u_{-10}+C u_{01}+C u_{0-1} \\
+\frac{b^{+}}{2} u_{11}+\frac{b^{+}}{2} u_{-1-1}-\frac{b^{-}}{2} u_{1-1}-\frac{b^{-}}{2} u_{-11}
\end{gathered}
$$

where $u_{i j}=u\left(T_{i j} P\right)$. This shows that $u_{00}=u(P)$ is less than a weighted average of values of $u$ at other points, hence $u(P)$ cannot be the maximal value of $u$. (This is the classical argument.)

If $L_{h}$ is not of positive type, we see from Propositions 4.1 and 4.2 that the inequality $L_{h} u(P)>0$ can be rewritten in the form

$$
\lambda_{0} \tilde{\Lambda}_{00}<\lambda_{1} \tilde{\Lambda}_{-\beta 0}+\lambda_{2} \tilde{\Lambda}_{-\beta-1}+\lambda_{3} \tilde{\Lambda}_{0-1}+\lambda u_{00}
$$

where $\tilde{\Lambda}_{i j}=\Lambda u\left(T_{i j} P\right) / \lambda$ and where $\lambda=\lambda_{0}-\lambda_{1}-\lambda_{2}-\lambda_{3}>0$. This shows that $\tilde{\Lambda}_{00}$ is less than a relevant weighted average and therefore cannot attain the maximum of $\Gamma u$. On the other hand, from (4.2b) we see that $u_{00}$ is a weighted average, namely

$$
\lambda_{0} u_{00}=\lambda_{1} u_{\beta 0}+\lambda_{2} u_{\beta 1}+\lambda_{3} u_{01}+\lambda \tilde{\Lambda}_{00},
$$

where a positive weight is being given to $\tilde{\Lambda}_{00}$, so that $u_{00}$ also cannot be a maximum of $\Gamma u$. Thus $\Gamma u(P)=\max \left\{u_{00}, \tilde{\Lambda}_{00}\right\}$ cannot be the maximum of $\Gamma u$. Q.E.D.

Remark 4.2. In a similar way, we can prove the above proposition with $\Gamma u$ replaced bu $\Gamma^{*} u$ :

$$
\begin{aligned}
\Gamma^{*} u(Q) & =u(Q), & & \text { if } L_{h} \text { is of positive type, } \\
& =\max \left\{u(Q), \Lambda^{*} u(Q) / \lambda\right\}, & & \text { otherwise. }
\end{aligned}
$$

Remark 4.3. Note from the proof that $\Gamma u_{00}$ is actually bounded by

$$
\max \left\{u_{\beta 0}, u_{\beta 1}, u_{01}, \tilde{\Lambda}_{-\beta 0}, \tilde{\Lambda}_{-\beta-1}, \tilde{\Lambda}_{0-1}\right\} .
$$

Similarly, $\Gamma^{*} u$ is actually bounded by $\max \left\{u_{-\beta 0}, u_{-\beta-1}, u_{0-1}, \tilde{\Lambda}^{*} u_{\beta 0}, \tilde{\Lambda}^{*} u_{\beta 1}, \tilde{\Lambda}^{*} u_{01}\right\}$. 
This observation is especially significant for points $P$ adjacent to a boundary because it shows what kind of boundary values enter into a priori estimates of $u$. For instance it shows that, if $0<b<(a c)^{1 / 2}$ and $L_{h} u>0$, one can estimate $u$ in the rectangle $\{(x, y):|x|<\bar{x},|y|<\bar{y}\}$ in terms of $\max _{P \in B} u(P)$ and $\max _{P \in B^{-}} \Lambda u(P)$ where

$$
\begin{aligned}
& B^{+}=\{(\bar{x}, y):-\bar{y}<y \leqq \bar{y}\} \cup\{(x, \bar{y}):-\bar{x}<x \leqq \bar{x}\}, \\
& B^{-}=\{(-\bar{x}, y):-\bar{y} \leqq y<\bar{y}\} \cup\{(x,-\bar{y}):-\bar{x} \leqq x<\bar{x}\}
\end{aligned}
$$

$(x, y, \bar{x}, \bar{y}$ being, of course, integral multiples of $h$ ).

4.2. Global Estimates and From-the-Boundary Estimates. In this section, we continue to discuss the operator (4.1), assuming it to be elliptic, but not of positive type. For simplicity, we assume that the boundary value problem is given on a mesh-lined domain, so that we do not have to specify different $L_{h}$ near the boundary. (This restriction is removed in Proposition 4.11 below.) The transition from the local maximum principle to global estimates is not as straightforward here as with positivetype operators, where simple use of a comparison function does the job. Straightforward use of Proposition 4.3 (with Remark 4.3 used near the boundary !) leads to an estimate of $\|u\|_{R_{h}}=\max _{P \in R_{h}}|u(P)|$ in terms of $\left\|L_{h} u\right\|_{R_{h}},\|u\|_{B_{h}}$, and $\|\Lambda u\|_{B_{h}^{\prime}}$, where $B_{h}^{\prime}$ and $B_{h}^{\prime \prime}$ are suitable portions of $B_{h}$. But in typical (Dirichlet) problems only the first two of these quantities are known, while $\Lambda u$ at the boundary is not known since it includes interior values of $u$.

One way to overcome this difficulty could be to modify the discrete formulation of the Dirichlet boundary condition on $B_{h}^{\prime \prime}$, by specifying there $\Lambda u / \lambda$ instead of $u$. (There may be boundary points which belong to both $B_{h}^{\prime}$ and $B_{h}^{\prime \prime}$; the boundary condition there should be taken differently on different occurrences!) This procedure is not very satisfactory, however, since it introduces $O(h)$ truncation errors. Another approach could be to formulate $L_{h}$ differently near the boundary, in such a way that global maximum-principles are obtained. It is possible to do so (see for example Section 4.4 below) with only $O\left(h^{2}\right)$ truncation errors. However, this entire approach of formulating the finite-difference equations so as to make their theoretical analysis easier seems unsatisfactory: One would like to analyze those (computationally convenient) difference schemes that are actually used in practice.

The following is a simple example that shows how to derive global estimates from the local maximum principle without reformulating the finite-difference equations.

Proposition 4.4. Let $R$ be a mesh-lined rectangle

$$
R=\{(x, y):|x|<\bar{x},|y|<\bar{y}\}, \text { where } \bar{x} / h, \bar{y} / h \text { are integers. }
$$

Assume $\left|L_{h} u\right| \leqq \bar{l}$ in $R_{h}$ and $|u| \leqq \bar{u}$ on the boundary $B_{h}$, where $\bar{l}, \bar{u}$ are constants. Then, for all $P \in R_{h}$,

$$
|u(P)| \leqq \frac{1}{2 c} \frac{\lambda_{0}}{\lambda}\left(\bar{y}+2 \frac{\lambda_{2}+\lambda_{3}}{\lambda} h\right) \bar{y} \bar{l}+\frac{\lambda_{0}^{2}+\left(\lambda_{0}-\lambda\right)^{2}}{\lambda^{2}} \bar{u} .
$$

Remark 4.4. A limiting case of the proposition is that of positive-type $L_{h}$. In that case (cf. Remark 4.1), $\lambda_{0} / \lambda=1$ and $\lambda_{i} / \lambda=0(i=1,2,3)$, so that (4.6) is reduced to

$$
|u(P)| \leqq \bar{y}^{2} \bar{l} / 2 c+\bar{u} .
$$

This is a classical, Gerschgorin-type result, which for $\bar{l}=0$ reproduces the global maximum principle. 
Proof. We can again take $b>0$. The comparison function $\psi(x, y)=(2 c)^{-1}$ $\left(\bar{y}^{2}-y^{2}\right) l$, where $l>\bar{l}$, is such that $L_{h}(u-\psi)=L_{h} u+l>0$, and hence, by Proposition 4.3 and Remark 4.2, the maximum of $\max \left\{\lambda(u-\psi), \Lambda(u-\psi), \Lambda^{*}(u-\psi)\right\}$ is attained on $B_{h}$. Similarly, the maximum of

$$
\max \left\{\lambda(-u-\psi), \Lambda(-u-\psi), \Lambda^{*}(-u-\psi)\right\}
$$

is also attained on $B_{h}$. With no loss of generality, we can assume that the maximum of these two maxima is $\left|\Lambda( \pm u-\psi)\left(P_{0}\right)\right|$ where $P_{0}=\left(-\bar{x}, y_{0}\right) \in B_{h}$. [The case where the maximum is $( \pm u-\psi)\left(P_{0}\right)$ is simpler. The case $P_{0}=\left(x_{0},-\bar{y}\right)$ is similar.] Thus

$$
\max \left\{|\lambda u(P)|,|\Lambda u(P)|,\left|\Lambda^{*} u(P)\right|\right\} \leqq\left|\Lambda u\left(P_{0}\right)\right|+\mu \quad\left(P \in R_{h}\right),
$$

where

$$
\mu=\max _{Q \in R_{h}} \Lambda \psi(Q)-\min _{Q \in B_{h}} \Lambda \psi(Q)=\frac{\lambda \bar{y}^{2}+2\left(\lambda_{2}+\lambda_{3}\right) \bar{y} h}{2 c} l .
$$

On the other hand,

$$
\begin{aligned}
\left|\Lambda u\left(P_{0}\right)\right|= & \mid \lambda_{0} u\left(-\bar{x}, y_{0}\right)-\lambda_{1} u\left(-\bar{x}+h, y_{0}\right) \\
& -\lambda_{2} u\left(-\bar{x}+h, y_{0}+h\right)-\lambda_{3} u\left(-\bar{x}, y_{0}+h\right) \mid \\
\leqq & \left(\lambda_{0}+\lambda_{3}\right) \bar{u}+\left(\lambda_{1}+\lambda_{2}\right) \hat{u}
\end{aligned}
$$

where

$$
\begin{aligned}
\hat{u} & =\max _{|y| \leqq \hat{y}}|u(-\bar{x}+h, y)|=|u(-\bar{x}+h, \hat{y})| \text { (say) } \\
& =\lambda_{0}^{-1}\left|\Lambda^{*} u(-\bar{x}+h, \hat{y})+\lambda_{1} u(-\bar{x}, \hat{y})+\lambda_{2} u(-\bar{x}, \hat{y}-h)+\lambda_{3} u(-\bar{x}+h, \hat{y}-h)\right| .
\end{aligned}
$$

Hence, by (4.7),

$$
\lambda_{0} \hat{u} \leqq\left|\Lambda u\left(P_{0}\right)\right|+\mu+\left(\lambda_{1}+\lambda_{2}\right) \bar{u}+\lambda_{3} \hat{u} .
$$

From (4.8) and (4.9), an estimate for $\left|\Lambda u\left(P_{0}\right)\right|$ in terms of $\bar{u}$ and $\mu$ is eliminated, plugged back into (4.7) and yields (4.6). Q.E.D.

A different approach, applicable to problems with Hölder continuous boundary data, is the following. First, using the local maximum principle (Proposition 4.3), one immediately gets an estimate of the form

$$
\left\|\left.u\right|_{\bar{R}_{h}} \leqq C_{1}\right\| L_{h} u\left\|_{R_{h}}+C_{2}\right\| u\left\|_{B_{h}}+C_{3}\right\| \Lambda u \|_{B_{h^{\prime}}}
$$

where the $C_{i}$ depend only on $R_{h}$ and $L_{h}$. For example, (4.7) is such an estimate, and it could be derived in the same way for any other mesh-lined domain contained in the strip $\{(x, y):|y| \leqq \bar{y}\}$. Then, for any $Q \in B_{h}^{\prime \prime}$, one can derive, again by Proposition 4.3 , an estimate of the type

$$
\max _{P \in \bar{R}_{h}} \frac{|u(P)-u(Q)|}{|P-Q|^{\alpha}} \leqq C_{4}|| L_{h} u\left\|_{R_{h}}+C_{5}\right\| u \|_{\bar{R}_{h}}
$$

where $|P-Q|$ denotes the distance between $P$ and $Q$, and where $0<\alpha \leqq 1$. Propositions 4.5 and 4.6 below are examples of such "from-the-boundary" estimates. From (4.11) one concludes

$$
\|\Lambda u\|_{B_{h^{\prime \prime}}} \leqq \lambda\|u\|_{B_{h}}+h^{\alpha}\left(C_{6}\left\|L_{h} u\right\|_{R_{h}}+C_{7}\|u\|_{\bar{R}_{h}}\right) .
$$


Finally, if $h$ is small, namely if $C_{3} C_{7} h^{\alpha}<1$, one can substitute (4.12) into (4.10) and get the desired estimate of $\|u\|_{R_{h}}$ in terms of $\left\|L_{h} u\right\|_{R_{h}}$ and $\|u\|_{B_{h}}$. For $h \rightarrow 0$, this estimate will be identical with a maximum-principle estimate for the continuous solution.

The derivation of (4.11), as well as the values of $C_{4}, C_{5}$ and $\alpha$, depend not only on $L$ and $R$, but also on $Q$. The following two propositions are basic examples.

Proposition 4.5. Let $R$ be any domain containing the rectangle

$$
R^{\prime}=\{(x, y):|x|<d, 0<y<d\} \quad(d / h \text { is an integer }),
$$

and such that its boundary $B$ contains the segment $B^{0}=\{(x, 0):|x|<d\}$. Let $h / d \leqq$ $2 \lambda / \alpha \lambda_{1}$. Suppose that, for all $P \in R_{h},\left|L_{h} u(P)\right|<\bar{l}$ and $|u(P)-u(O)|<\bar{u}$. Suppose further that

$$
|u(Q)-u(O)| /|Q-O|^{\alpha} \leqq H \quad\left(Q \in B_{h}\right),
$$

where $|Q-O|$ is the distance from $Q$ to the origin $O=(0,0)$. Then

$$
\frac{|u(P)-u(O)|}{|P-O|^{\alpha}} \leqq \frac{a+6|b|+2 c}{(1-\alpha) c} \max \left\{H, \frac{2 \lambda_{0}-\lambda}{\lambda} \frac{\bar{u}}{d^{\alpha}}\right\}+\frac{d^{2-\alpha}}{\alpha(1-\alpha) c} \bar{l}
$$

for all $P \in R_{h}$.

Proof. With no loss of generality we can assume that $b \geqq 0$ and that $u(O)=0$. Our comparison function will be $\psi=A r^{\alpha}+C y^{\alpha}$, where $r^{2}=x^{2}+y^{2}$ and where the constants $A$ and $C$ are selected (see below) so that

$$
\begin{aligned}
L_{h} \psi(P) & <-\bar{l}, & & \text { for } P \in R_{h}^{\prime}, \\
|\psi(x, 0)| & \geqq H|x|^{\alpha}, & & \text { for }(x, 0) \in B_{h}^{0}, \\
|\psi(P)| & \geqq \bar{u}, & & \text { for } P \in B_{h}^{1}, \\
\Lambda^{*} \psi(P) & \geqq\left(2 \lambda_{0}-\lambda\right) \bar{u} \geqq\left|\Lambda^{*} u\right|, & & \text { for } P \in B_{h}^{2} \cup B_{h}^{3} .
\end{aligned}
$$

Here $B^{1}=\{(-d, y): 0 \leqq y \leqq d\}, B^{2}=\{(x, d):|x| \leqq d\}$ and $B^{3}=\{(d, y): 0 \leqq$ $y \leqq d\}$. From (4.14) we will have $L_{h}( \pm u-\psi)>0$, and hence, by Proposition 4.3 (actually by Remark 4.2, with Remark 4.3 applied at points adjacent to $B_{h}^{\prime}=\bigcup_{0}^{3} B_{h}^{i}$ ), it will follow that $( \pm u-\psi)$ is bounded in $R_{h}^{\prime}$ by its values on $B_{h}^{0} \cup B_{h}^{1}$ and by the values of $\Lambda^{*}( \pm u-\psi) / \lambda$ on $B_{h}^{2} \cup B_{h}^{3}$. But these values will be nonpositive by (4.15), and therefore $( \pm u-\psi)$ must be nonpositive in $R_{h}^{\prime}$. Hence $|u| \leqq \psi$ in $R_{h}^{\prime}$, which will prove the proposition.

To choose $A$ and $C$ so that (4.14) and (4.15) are satisfied, we need some simple inequalities for difference-quotients of $r^{\alpha}$ and $y^{\alpha}$. First, we claim that, in $R_{h}^{\prime}$,

$$
\partial_{r r} r^{\alpha} \leqq \partial_{s s} r^{\alpha} \leqq \alpha r^{\alpha-2} \quad(s=x \text { or } y),
$$

where $\partial_{r} r^{\alpha}=\left[(r+h)^{\alpha}-2 r^{\alpha}+(r-h)^{\alpha}\right] / h^{2}$. Indeed,

$$
\partial_{s s} r^{\alpha}=\left[\left(r^{2}+2 s h+h^{2}\right)^{\alpha / 2}-2 r^{\alpha}+\left(r^{2}-2 s h+h^{2}\right)^{\alpha / 2}\right] / h^{2} \equiv \phi(r, s), \text { say. }
$$

Since sgn $\partial \phi / \partial s=\operatorname{sgn}(-s)$, we clearly get $\partial_{r_{r}} r^{\alpha}=\phi(r, r) \leqq \phi(r, s) \leqq \phi(r, 0)$, while a 3-term Taylor formula shows that $\phi(r, 0) \leqq \alpha r^{\alpha-2}$, establishing (4.16a). A similar argument, with $s=x \pm y$, yields

$$
2^{1 / 2} \partial_{r r} r^{\alpha} \leqq \partial_{x \pm y, x \pm y} r^{\alpha} \equiv\left(T_{1 \pm 1}-2 T_{00}+T_{-1 \mp 1}\right) r^{\alpha} / 2 h^{2} \leqq \alpha r^{\alpha-2}
$$


Next we note that

$$
\partial_{r r} r^{\alpha} \equiv \phi(x, y) \geqq \phi(0, y)=\partial_{y y} y^{\alpha},
$$

since sgn $\partial \bar{\phi} / \partial x=\operatorname{sgn} x$. Also, by a 5-term Taylor formula,

$$
\partial_{y y} y^{\alpha} \leqq-\alpha(1-\alpha) y^{\alpha-2} \leqq-\alpha(1-\alpha) r^{\alpha-2} .
$$

From (4.16a-d) we then get

$$
\left|\partial_{x y}^{ \pm} r^{\alpha}\right|=\left|\partial_{x \pm y, x \pm y} r^{\alpha}-\frac{1}{2} \partial_{x x} r^{\alpha}-\frac{1}{2} \partial_{y y} r^{\alpha}\right| \leqq-\frac{3}{1-\alpha} \partial_{y y} y^{\alpha}
$$

Next, using $O(h)$ Taylor expansions with the assumption $h / d \leqq 2 \lambda / \alpha \lambda_{1}$, we can easily see that

$$
\begin{array}{lll}
\Lambda^{*} r^{\alpha} \geqq 0, & \Lambda^{*} y^{\alpha} \geqq \lambda d^{\alpha} & \left((x, y) \in B^{2}\right), \\
\Lambda^{*} r^{\alpha} \geqq \lambda d^{\alpha}, & \Lambda^{*} y^{\alpha} \geqq 0 & \left((x, y) \in B^{3}\right) .
\end{array}
$$

Now, from (4.16) it follows that

$$
L_{h} \psi \leqq\left[c C-\frac{A}{1-\alpha}(a+6 b+c)\right] \partial_{y y} y^{\alpha},
$$

and therefore, by (4.16d), we can satisfy (4.14) by taking

$$
C=\frac{a+6 b+c}{(1-\alpha) c} A+\frac{i d^{2-\alpha}}{\alpha(1-\alpha) c} .
$$

Then, from (4.16f, $\mathrm{g}$ ) it follows that (4.15) will be satisfied by taking

$$
A=\max \left\{H, \frac{2 \lambda_{0}-\lambda}{\lambda} \frac{\bar{u}}{d^{\alpha}}\right\} .
$$

Thus, with these values of $A$ and $C$, we have $|u(x, y)| \leqq \psi(x, y)$, and hence $|u(x, y)| / r^{\alpha}$ $\leqq A+C$, for all $(x, y) \in R_{h}^{\prime}$. This proves (4.13) for $P \in R_{h}^{\prime}$. For $P \notin R_{h}^{\prime}$, (4.13) is trivially satisfied, since $|P-O|>d$. Q.E.D.

Remark 4.5. The provision $h / d \leqq 2 \lambda / \alpha \lambda_{1}$ is not necessary. Without this provision the first inequality in (4.16f) may be violated, but (4.15c) can be satisfied by adding a term $E=A d^{\alpha}\left(\lambda_{1} \alpha h / 2 d-\lambda\right)$ to the comparison function $\psi$, which will introduce an additional term $E / h^{\alpha} \leqq O\left(h^{1-\alpha}\right)$ to the estimate (4.13).

In the above proof we made a clear use of Remark 4.3, owing to which information concerning $\Lambda^{*} u(P)$ was needed only on $B^{2} \cup B^{3}$, but not on $B^{0}$. If $\Lambda^{*} \psi \geqq \pm \Lambda^{*} u$ on $B^{0}$ were to be required, it would not be possible to have $\psi=O\left(r^{\alpha}\right)$. In the next example, dealing with a corner boundary point, the situation is more complicated, since, whether $\Gamma$ or $\Gamma^{*}$ are used, one cannot escape having to know either $\Lambda u$ or $\Lambda^{*} u$ at boundary points near the corner. The only solution is to use both $\Gamma$ and $\Gamma^{*}$, that is, a joint use of Proposition 4.3 and Remark 4.2. To display clearly the features of the situation, we shall avoid other complications by assuming the comparison function $\psi$ to be given. (But see Remark 4.7.)

Proposition 4.6. Let $L_{h}$ be the elliptic operator (4.1), with $0<b<(a c)^{1 / 2}$. Let $R$ be any domain with boundary $B$ such that

$$
R \supset R^{1} \cup R^{2} \text { and } B \supset B^{1} \cup B^{2} \text {, }
$$


where

$$
\begin{aligned}
& R^{1}=\{(x, y):-d<x<0,-d<y \leqq-x\}, \\
& R^{2}=\{(x, y): 0<y<d,-y \leqq x<d\}, \\
& B^{1}=\{(0, y):-d \leqq y \leqq 0\}, \\
& B^{2}=\{(x, 0): 0 \leqq x \leqq d\},
\end{aligned}
$$

$d$ being some integral multiple of $h$. Suppose

$$
\begin{aligned}
\left|L_{h} u(P)\right| & <-L_{h} \psi(P), & & \text { for } P \in R_{h}, \\
|u(P)-u(O)| & \leqq \psi(P), & & \text { for } P \in B_{h}, \\
|\Gamma u(P)-u(O)| & \leqq \Gamma \psi(P), & & \text { for } P \in B_{h}^{3}, \\
\left|\Gamma^{*} u(P)-u(O)\right| & \leqq \Gamma^{*} \psi(P), & & \text { for } P \in B_{h}^{4},
\end{aligned}
$$

where $O=(0,0)$, and

$$
\begin{aligned}
& B^{3}=\{(-d, y):|y| \leqq d\} \cup\{(x,-d):-d \leqq x \leqq 0\}, \\
& B^{4}=\{(d, y): 0 \leqq y \leqq d\} \cup\{(x, d):|x| \leqq d\} .
\end{aligned}
$$

Then

$$
|u(P)-u(O)| \leqq \psi(P), \quad \text { for } P \in\left(R^{1} \cup R^{2}\right)_{h} .
$$

Remark 4.6. For points $P \notin R_{h}^{1} \cup R_{h}^{2}$, we have $|P-O|>d$ and, therefore, an estimate of the form (4.11) is trivially obtainable. For points $P \in R_{h}^{1} \cup R_{h}^{2}$, the estimate (4.19) is of the form (4.11), provided $\psi(x, y)=O\left(r^{\alpha}\right)$. Such a function $\psi$, with a suitable value of $\alpha$, can be constructed for $u$ with Hölder-continuous boundary conditions, because the requirement we impose on $\psi$ in the vicinity of the origin $O$ involves $u$ only, whereas $\Gamma u$ and $\Gamma^{*} u$ enter the requirements only at points $P$ bounded away from $O$, where the precision of the requirements is not important. For instance, $(4.18 \mathrm{~b})$ can be replaced by

$\left(4.18 b^{\prime}\right)$

$$
\left(2 \lambda_{0}-\lambda+1\right) \max |u| \leqq \Gamma \psi(P) \quad\left(P \in B_{h}^{3}\right),
$$

which is a stronger requirement but still allows construction (cf. Remark 4.7) of $\psi=O\left(r^{\alpha}\right)$.

Proof. Let $\phi(P)=u(P)-u(O)-\psi(P)$. Denote

$$
\begin{aligned}
\Gamma^{\prime} \phi(P) & =\phi(P), & & \text { if } P \in B_{h}^{1} \cup B_{h}^{2}, \\
& =\Gamma \phi(P), & & \text { if } P \in R_{h}^{1} \cup B_{h}^{3}, \\
& =\Gamma^{*} \phi(P), & & \text { if } P \in R_{h}^{2} \cup B_{h}^{4} .
\end{aligned}
$$

By (4.17), $L_{h} \phi(P)>0$ for all $P \in R_{h}^{1} \cup R_{h}^{2}$, and therefore, by Proposition 4.3 and Remarks 4.2 and 4.3, $\Gamma^{\prime} \phi(P)$ must attain its maximum on the boundary of $R^{1} \cup R^{2}$, i.e., on $B_{h}^{1} \cup B_{h}^{2} \cup B_{h}^{3} \cup B_{h}^{4}$. (Remark 4.3 is applied here on $R_{h}^{1} \cap R_{h}^{2}$ as well as near $B_{h}^{1} \cup B_{h}^{2}$.) By (4.18), that maximum is nonnegative, and therefore $\phi(P) \leqq 0$ throughout $R_{h}^{1} \cup R_{h}^{2}$. This and a similar result with $-u$ replacing $u$ together imply (4.19). Q.E.D.

Remark 4.7. The actual construction of a comparison function $\psi$ that satisfies (4.17)-(4.18) is straightforward, though cumbersome. First one constructs a continuous 
function $\psi$ that satisfies the continuous analog of the requirements. This is easy, because, by a linear transformation, (4.17) is transformed into a requirement on $\Delta \psi(\xi, \eta)$, which can be satisfied, together with the transformed boundary requirements, by a function $\psi$ of the form

$$
\psi=A_{1}\left(\xi^{2}+\eta^{2}\right)^{\alpha_{1}}+A_{2}\left(\xi^{2}+\eta^{2}\right)^{\alpha_{2}} \sin (\beta \theta+\gamma), \text { where } \tan \theta=\eta / \xi .
$$

This function, possibly with slightly larger coefficient $A_{2}$, can then be seen to satisfy (4.17)-(4.18), for sufficiently small $h$.

4.3. Interior and Uniform Estimates of Difference-Quotients. For positive-type operators, estimates of difference-quotients are obtained in exactly the same way as for the corresponding continuous, uniformly-elliptic operators. Cf. Brandt [7], [8], [9]. Similar though more laborious methods can be applied to general (not necessarily positive-type) operators like (4.1), using decompositions of the type (4.2). An example:

Proposition 4.7. Let

$$
L_{h}=a \partial_{x x}+b^{+} \partial_{x y}^{+}+b^{-} \partial_{x y}^{-}+c \partial_{y y}=\frac{\lambda^{2}-\Lambda^{*} \Lambda}{h^{2}}+\mu \partial_{y y}
$$

where $\Lambda, \Lambda^{*}$ and $\lambda>0$ are as in (4.2), with nonnegative $\lambda_{0}, \lambda_{1}, \lambda_{2}, \lambda_{3}$, and where $0<$ $\mu \leqq \lambda^{2} / 2$. Assume

$$
\left|L_{h} u(P)\right|<\bar{l}, \quad|u(P)| \leqq \bar{u} \quad\left(P \in R_{h}\right),
$$

where

$$
R \supset\{(x, y):|x| \leqq d,|y| \leqq 2 d\} .
$$

Then

$$
\left|\partial_{y} u(0,0)\right| \leqq \frac{d}{2 \mu} \bar{l}+\frac{2 \lambda_{0}-\lambda}{\lambda}\left[\frac{a+c}{\mu}+2 \frac{\left|\lambda_{1}-\lambda_{3}\right|}{\lambda}+\frac{h}{d}\right] \frac{\bar{u}}{d} .
$$

Remark 4.8. The decomposition in (4.20) exists whenever $L_{h}$ is elliptic but not of positive type, provided $b^{+} b^{-} \geqq 0$. Indeed, a sufficiently small $\mu$ can be chosen so that the operator $L_{h}-\mu \partial_{y y}$ is still elliptic and Propositions 4.1-4.2 can be applied to it.

Proof. As in the proof of Proposition 3.5, we view the difference

$$
\hat{u}(x, y, z)=\frac{1}{2}[u(x, y+z)-u(x, y-z)]
$$

as a function of the three variables $(x, y, z)$, defined on

$$
\hat{R}_{h}=\left\{(x, y, z): \frac{x}{h}, \frac{y}{h}, \frac{z}{h} \text { are integers; }|x|,|y|<d, 0<z<d\right\},
$$

and we introduce the three-dimensional operator

$$
\hat{L}_{h}=a \partial_{x x}+b^{+} \partial_{x y}^{+}+b^{-} \partial_{x y}^{-}+(c-\mu) \partial_{y y}+\mu \partial_{z z}=\frac{\lambda^{2}-\Lambda^{*} \Lambda}{h^{2}}+\mu \partial_{z z},
$$

for which we have

$$
\left|\hat{L}_{h} \hat{u}(x, y, z)\right|=\frac{1}{2}\left|L_{h} u(x, y+z)-L_{h} u(x, y-z)\right|<\bar{l} \quad\left((x, y, z) \in \hat{R}_{h}\right) .
$$

The comparison function will have the form 


$$
\psi(x, y, z)=\frac{\bar{l}}{2 \mu}\left(d z-z^{2}\right)+A_{1}\left[x^{2}+y^{2}+z^{2}+\frac{a+c}{\mu}\left(d z-z^{2}\right)+A_{2} h\right],
$$

where $A_{1}, A_{2}$ are constants to be determined later in such a way that $\psi$ will suitably dominate $\hat{u}$ on the boundary of $\hat{R}_{h}$. This form of $\psi$ immediately implies $L_{h} \psi=-\bar{l}$, so that by writing $\phi=\hat{u}-\psi$ it follows that

$$
\hat{L}_{h} \phi(x, y, z)>0 \quad\left((x, y, z) \in \hat{R}_{h}\right) .
$$

We now observe that $\hat{L}_{h}$ satisfies a generalized maximum principle similar to Proposition 4.3. Indeed, if we put (for $b^{ \pm} \geqq 0$, e.g.)

$$
\begin{aligned}
& \tilde{\Lambda} \phi(x, y, z)=\frac{1}{\lambda} \Lambda \phi(x, y, z) \\
& \quad=\frac{1}{\lambda}\left\{\lambda_{0} \phi(x, y, z)-\lambda_{1} \phi(x+h, y, z)-\lambda_{2} \phi(x+h, y+h, z)-\lambda_{3} \phi(x, y+h, z)\right\},
\end{aligned}
$$

and if for any fixed point $(x, y, z) \in \hat{R}_{h}$ we denote

$$
\phi_{i j k}=\phi(x+i h, y+j h, z+k h), \quad \tilde{\Lambda}_{i j k}=\tilde{\Lambda} \phi(x+i h, y+j h, z+k h),
$$

then it can be seen from (4.22) that (4.23) is equivalent to

$$
\lambda_{0} \tilde{\Lambda}_{000}<\lambda_{1} \tilde{\Lambda}_{-100}+\lambda_{2} \tilde{\Lambda}_{-1-10}+\lambda_{3} \tilde{\Lambda}_{0-10}+\frac{\lambda^{2}-2 \mu}{\lambda} \phi_{000}+\frac{\mu}{\lambda}\left(\phi_{001}+\phi_{00-1}\right) .
$$

The definition of $\tilde{\Lambda}$ can be rewritten as

$$
\lambda_{0} \phi_{000}=\lambda_{1} \phi_{100}+\lambda_{2} \phi_{110}+\lambda_{3} \phi_{010}+\lambda \tilde{\Lambda}_{000} .
$$

These relations clearly show that both $\phi$ and $\tilde{\Lambda} \phi$ at $(x, y, z)$ are less than weighted averages of $\phi$ and $\widetilde{\Lambda} \phi$ at other points. Thus $\max \{\phi, \widetilde{\Lambda} \phi\}$ in $\hat{R}_{h}$ must be less than its maximum on the boundary of $\hat{R}_{h}$, i.e., on

$$
\hat{B}_{h}=B_{h}^{1} \cup B_{h}^{2} \cup B_{h}^{3} \cup B_{h}^{4} \cup B_{h}^{5} \cup B_{h}^{6},
$$

where

$$
\begin{array}{ll}
B^{1}=\{x=-d,-d \leqq y<d, 0<z<d\}, & B^{2}=\{x=d,|y| \leqq d, 0 \leqq z \leqq d\}, \\
B^{3}=\{-d \leqq x<d, y=-d, 0<z<d\}, & B^{4}=\{|x| \leqq d, y=d, 0 \leqq z \leqq d\}, \\
B^{5}=\{|x| \leqq d,|y| \leqq d, z=0\}, & B^{6}=\{|x| \leqq d,|y| \leqq d, z=d\} .
\end{array}
$$

Closer examination of (4.24)-(4.25) at net-points adjacent to $\widehat{B}_{h}$ shows (as in Remark 4.5) that $\phi$ and $\tilde{\Lambda} \phi$ in $\hat{R}_{h}$ must in fact be less than either the maximum of $\phi$ on $B_{h}^{2} \cup$ $B_{h}^{4} \cup B_{h}^{5} \cup B_{h}^{6}$ or the maximum of $\tilde{\Lambda} \phi$ on $B_{h}^{1} \cup B_{h}^{3}$. Both these maxima can be made negative by choosing

$$
A_{1}=\frac{\lambda_{0}+\lambda_{1}+\lambda_{2}+\lambda_{3}}{\lambda} \frac{\bar{u}}{d^{2}}, \quad A_{2}=2 d\left|\lambda_{1}-\lambda_{3}\right| / \lambda
$$

(the term with $A_{2}$ being designed so that $\Lambda\left(x^{2}+y^{2}+A_{2} h\right) \geqq \lambda\left(x^{2}+y^{2}\right)$ on $\left.B_{h}^{1} \cup B_{h}^{3}\right)$. Therefore, for these values of $A_{1}$ and $A_{2}, \phi$ must be negative throughout $\hat{R}_{h}$. Hence $\hat{u}(x, y, z) \leqq \psi(x, y, z)$ in $\hat{R}_{h}$, and in particular 


$$
\partial_{\nu} u(0,0)=\frac{1}{h} \hat{u}(0,0, h) \leqq \frac{1}{h} \psi(0,0, h) \leqq \frac{d}{2 \mu} \bar{l}+A_{1}\left[\frac{a+c}{\mu} d+A_{2}+h\right] .
$$

This implies (4.21). Q.E.D.

The estimate in Proposition 4.7 blows up near the boundary: If $d_{p}$ denotes the distance $|P-Q|$ from the point of estimate $P$ (conveniently taken above as the origin) to the nearest boundary point $Q$, then $d \leqq d_{p}$ and therefore for $d_{p} \rightarrow 0$ the right-hand side of (4.21) grows to infinity. To get uniform estimates, we should have $\bar{u}$ in (4.21) proportional to $d$. Now, for $\bar{u}$ in the proposition we could actually take

$$
\bar{u}=\sup _{P^{\prime} \in R^{\prime}}\left|u\left(P^{\prime}\right)-u(Q)\right|, \quad R^{\prime}=\left\{P^{\prime}:\left|P-P^{\prime}\right|<3 d\right\},
$$

where $u(Q)$ is a constant and therefore its subtraction from $u$ cannot affect estimates of difference-quotients. Furthermore, $d$ in the proposition can be chosen so that $3 d \geqq d_{p}$, and then any $P^{\prime} \in R^{\prime}$ satisfies $\left|P^{\prime}-Q\right| \leqq 3 d+d_{p} \leqq 6 d$, and hence

$$
\bar{u} \leqq \sup _{\left|P^{\prime}-Q\right|<\beta d}\left|u\left(P^{\prime}\right)-u(Q)\right| \leqq 6 d \sup \frac{\left|u\left(P^{\prime}\right)-u(Q)\right|}{\left|P^{\prime}-Q\right|}
$$

Plugging this into (4.21) gives a uniform estimate for $\left|\partial_{y} u\right|$, as desired, provided we have a uniform estimate for the difference-quotient in the right-hand side of (4.26): In that difference-quotient $Q$ is a boundary point, hence its estimate is what we called a "from-the-boundary" estimate, examples of which were given before (Section 4.2).

We thus see that uniform estimates can be derived by combining interior estimates, such as (4.21), with from-the-boundary estimates, such as (4.11).

4.4. Variable Coefficients and Lower-Order Terms. So far, we have dealt with the homogeneous elliptic operator (4.1) with constant coefficients only. We shall see in this section that essentially the same methods work for operators with variable coefficients and lower-order terms. Only mild restrictions will be needed on the variability of the principal coefficients and on the magnitude of the others. The restrictions are mild in the sense that they disappear in the continuous limit, i.e., they are automatically satisfied for sufficiently small mesh-sizes.

Let us first consider the operator (4.1) with variable coefficients $a, b^{+}, b^{-}, c$, where, for simplicity,

$$
0<\left(b^{+}+b^{-}\right) / 2=b<(a c)^{1 / 2}, \quad b^{+} \geqq 0, b^{-} \geqq 0 .
$$

Propositions 4.1 (with $\beta=1$ ) and 4.2 still hold, except that now the nonnegative decomposition coefficients $\lambda_{0}, \lambda_{1}, \lambda_{2}, \lambda_{3}$ and $\lambda$ are all variable; i.e., at each point the decomposition is done with different $\lambda_{i}$ 's. Since one can always multiply both sides of the equation $L_{h} u=f$ by an arbitrary function, we have to normalize $L_{h}$ in some way. A convenient normalization here is to take $\lambda_{0} \equiv 1$, i.e., to replace $\lambda_{i}$ and $\lambda$ by $\lambda_{i} / \lambda_{0}$ and $\lambda / \lambda_{0}$, respectively. Consequently, we have, for any net-point $Q$,

$$
\begin{aligned}
& \Lambda(Q)=T_{00}-\lambda_{1}(Q) T_{10}-\lambda_{2}(Q) T_{11}-\lambda_{3}(Q) T_{01}, \\
& \lambda(Q)=1-\lambda_{1}(Q)-\lambda_{2}(Q)-\lambda_{3}(Q)<1 .
\end{aligned}
$$

Introducing the operators

$$
\hat{\Lambda}(Q)=\Lambda(Q)-\lambda(Q)^{2} T_{00}, \quad \hat{\lambda}(Q)=\lambda(Q)-\lambda(Q)^{2},
$$




$$
\hat{\Gamma} u(Q)=\max \{u(Q), \hat{\Lambda}(Q) u(Q) / \hat{\lambda}(Q)\},
$$

we can then state the following generalized local maximum principle.

Proposition 4.8. Let $L_{h}$ be the difference operator (4.1), satisfying (4.27) and (4.2), with $\lambda_{0} \equiv 1$ and with the variability of its coefficients being restricted so that

$$
\lambda_{k}\left(T_{\alpha \gamma} Q\right) \leqq \frac{\lambda_{k}(Q)}{1-\lambda(Q)^{2}} \quad(0 \leqq \alpha, \gamma \leqq 1 ; k=1,2,3) .
$$

If $L_{h} u(P)>0$ then $\hat{\Gamma} u($ defined by (4.29)) cannot attain its maximum at $P$.

Proof. By (4.2), $h^{2} L_{h} u(P)>0$ can be rewritten in the form

$$
\begin{aligned}
\hat{\Lambda}(P) u(P) & <\sum_{i=1}^{3} \lambda_{i}(P) \Lambda(P) u\left(P_{i}\right) \\
& =\sum_{i=1}^{3} \frac{\lambda_{i}(P)}{1-\lambda\left(P_{i}\right)^{2}} \hat{\Lambda}\left(P_{i}\right) u\left(P_{i}\right)+\sum_{i=1}^{3} \lambda_{i}(P)\left[\Lambda(P)-\frac{\hat{\Lambda}\left(P_{i}\right)}{1-\lambda\left(P_{i}\right)^{2}}\right] u\left(P_{i}\right)
\end{aligned}
$$

where $P_{1}=T_{-10} P, P_{2}=T_{-1-1} P, P_{3}=T_{0-1} P$. By (4.29a) and (4.30), each of the bracketed operators in the last summation has only nonnegative weights. Hence $\hat{\Lambda}(P) u(P) / \hat{\lambda}(P)$ is less than a weighted average of values of $\hat{\Gamma} u$ at $P$ and at neighboring points and therefore it is less than max $\hat{\Gamma} u$. Furthermore, by (4.29a),

$$
\left[1-\lambda(P)^{2}\right] u(P)=\hat{\Lambda}(P) u(P)+\lambda_{1}(P) u\left(T_{10} P\right)+\lambda_{2}(P) u\left(T_{11} P\right)+\lambda_{3}(P) u\left(T_{01} P\right),
$$

which shows that $u(P)$ is a weighted average of $\hat{\Lambda}(P) u(P) / \hat{\lambda}(P)$ and values of $u$ at neighboring points, so that $u(P)$ is also less than $\max \hat{\Gamma} u$. Q.E.D.

Note that (4.30) is a mild restriction. It allows $\lambda_{k}$ to have jumps whose magnitudes do not depend on $h$, so that arbitrary jumps are allowed when $h \rightarrow 0$. The proposition, however, is still too restrictive since we assumed $b>0$. In contrast, the sign of $b$ can freely vary in Propositions $3.2^{\prime}, 3.3^{\prime}$ and $3.4^{\prime}$, where the restriction is of the type (3.16). Thus, to get a more general result we have to combine the technique of Proposition 4.8 with that of Section 3.3 . One way of doing that is to write $L_{h}$ in the form

$$
L_{h}=\left(\lambda^{2}-\Lambda^{*} \Lambda\right) / h^{2}+\mu \Delta_{h}, \quad \lambda_{0}=1, \quad \lambda^{2}=5 \mu>0,
$$

where $\Lambda, \lambda_{0}$ and $\lambda$ are as in (4.2), and $\Delta_{h}=\partial_{x x}+\partial_{y y}$ is the discrete Laplacian. We first have to show that (4.31) is sufficiently general.

Proposition 4.9. If $L_{h}$ is elliptic and of the form (4.1), then, normalized by $a$ suitable scalar multiplier, $L_{h}$ can be written in the form (4.31).

Proof. If $L_{h}$ is not of positive type, then $L_{h}(\mu)=L_{h}-\mu \Delta_{h}$ is also not of positive type, for all $\mu \geqq 0$. There clearly exists $\mu_{1}$ such that, for all $0 \leqq \mu<\mu_{1}$, the operator $L_{h}(\mu)$ is still elliptic, and therefore can be decomposed as in (4.2) with $\lambda=\lambda(\mu)>0$, and such that $L_{h}\left(\mu_{1}\right)$ is parabolic, i.e., $a c-b^{2}=0$, so that, by Proposition 4.2, $\lambda \searrow 0$ as $\mu \nearrow \mu_{1}$. Thus $\lambda^{2}-5 \mu$ is positive for $\mu=0$ and negative for $\mu \rightarrow \mu_{1}$, and hence must vanish for some intermediate $\mu$. For that value of $\mu, \lambda_{0}^{-2} L_{h}$ is of the form (4.31).

If $L_{h}$ is of positive type, then there exists $\mu_{0} \geqq 0$ is such that $L_{h}\left(\mu_{0}\right)$ is still of positive type, but $L_{h}(\mu)$ is not of positive type for $\mu>\mu_{0}$. Clearly, $L_{h}\left(\mu_{0}\right)$ is either elliptic $\left(a>0, a c>b^{2}\right)$ or parabolic $\left(a+c \geqq 0, a c=b^{2}\right)$. If $L_{h}\left(\mu_{0}\right)$ is elliptic, then there exists $\mu_{1}>\mu_{0}$ such that $L_{h}\left(\mu_{1}\right)$ is parabolic, and therefore $\lambda^{2}-5 \mu$ is negative for 
$\mu \nearrow \mu_{1}$. For $\mu \searrow \mu_{0}$, by Remark 4.1, $\lambda \rightarrow \infty$ and therefore $\lambda^{2}-5 \mu>0$. Thus, for some intermediate $\mu, \lambda^{2}-5 \mu$ vanishes and $\lambda_{0}^{-2} L_{h}$ is of the form (4.31).

If $L_{h}\left(\mu_{0}\right)$ is parabolic, then, since it is of positive type, it must be proportional to either $\partial_{x x}$, or $\partial_{y y}$, or $\left(\partial_{x}^{+}+\partial_{y}^{-}\right)\left(\partial_{x}^{-}+\partial_{y}^{+}\right)$, or $\left(\partial_{x}^{+}+\partial_{y}^{+}\right)\left(\partial_{x}^{-}+\partial_{y}^{-}\right)$; in particular, it may vanish. In either case, $L_{h}\left(\mu_{0}\right)=\lambda^{2}-\Lambda^{*} \Lambda$, where $\Lambda$ is as in (4.2), but with at least two of $\left\{\lambda_{1}, \lambda_{2}, \lambda_{3}\right\}$ vanishing and with arbitrary $\lambda$. For example, if $L_{h}\left(\mu_{0}\right)=a \partial_{x x}$, then $\Lambda=\lambda_{0} T_{00}-\lambda_{1} T_{01}$, where $\lambda_{0}$ and $\lambda_{1}$ are any positive numbers satisfying $\lambda_{0} \lambda_{1}=a$, $\lambda_{0}>\lambda_{1}$. Clearly, we can choose $\lambda_{0}$ so that $\lambda^{2}=\left(\lambda_{0}-\lambda_{1}\right)^{2}=5 \mu_{0}$, and then $\lambda_{0}^{-2} L_{h}$ is of the form (4.31). Q.E.D.

In the variable-coefficients case, $\lambda_{1}, \lambda_{2}, \lambda_{3}, \lambda$ and $\mu$ are of course variable too. If $L_{h}$ is uniformly elliptic, then $\mu$ is bounded away from zero. For compatibility of the notation for the two cases $\beta=1$ and $\beta=-1$ (cf. (4.2)), we write in the latter case, $\lambda_{5}$ and $\lambda_{4}$ instead of $\lambda_{1}$ and $\lambda_{2}$, respectively. Thus, the elliptic operator $L_{h}$, including now lower-order terms too, has, at any net-point $Q$, the general form

$$
\begin{aligned}
L_{h}(Q) & =\frac{\lambda(Q)^{2}-\Lambda^{*}(Q) \Lambda(Q)}{h^{2}}+\mu(Q) \Delta_{h}+r(Q) \partial_{x}+s(Q) \partial_{\nu}-q(Q), \\
\Lambda(Q) & =1-\sum_{i=1}^{5} \lambda_{i}(Q) T_{i}, \quad \lambda(Q)=1-\sum_{1}^{5} \lambda_{i}(Q), \quad \mu(Q)=\lambda^{2}(Q) / 5, \\
T_{1} & =T_{10}, \quad T_{2}=T_{11}, \quad T_{3}=T_{01}, \quad T_{4}=T_{-11}, \quad T_{5}=T_{-10},
\end{aligned}
$$

where either $\lambda_{1}(Q)=\lambda_{2}(Q)=0$ or $\lambda_{4}(Q)=\lambda_{5}(Q)=0$, and where $\lambda_{i} \geqq 0, \lambda \geqq 0$.

With the auxiliary operators being now defined as

$$
\begin{gathered}
\hat{\Lambda}(Q)=\Lambda(Q)-\frac{1}{2} \mu(Q)\left(T_{10}+T_{01}+T_{-10}+T_{0-1}\right), \hat{\lambda}(Q)=\lambda(Q)-2 \mu(Q), \\
\hat{\Gamma} u(Q)=\max \{u(Q), \hat{\Lambda}(Q) u(Q) / \hat{\lambda}(Q)\}
\end{gathered}
$$

the more general local maximum principle reads as follows:

Proposition 4.10. Let $L_{h}$, of the form (4.32) be such that

$$
\begin{aligned}
\mid \lambda_{i}(Q)- & \lambda_{i}\left(T_{\alpha \gamma} Q\right) \mid \leqq \frac{1}{3} \mu(Q) \quad(-1 \leqq \alpha, \gamma \leqq 1,1 \leqq i \leqq 5), \\
& \max \left\{3 h|r(Q)|, 3 h|s(Q)|, \frac{3}{2} h^{2} q(Q)\right\} \leqq \mu(Q) .
\end{aligned}
$$

If $L_{h} u(P)>0$ and $q(P) \geqq 0$, then $\hat{\Gamma} u$ (defined by (4.33)) cannot attain a positive maximum at $P$.

Proof. Using (4.32)-(4.33) we rewrite the inequality $L_{h} u(P)>0$ in the form

$$
\hat{\Lambda}(P) u(P)<\sum_{i=1}^{5} \lambda_{i}(P) \hat{\Lambda}\left(P_{i}\right) u\left(P_{i}\right)+\sum_{\alpha, \gamma=-2}^{2} \omega_{\alpha \gamma} u\left(T_{\alpha \gamma} P\right)
$$

where $T_{i} P_{i}=P$, and $\omega_{\alpha \gamma}$ are coefficients that can be explicitly computed in terms of $\lambda_{k}\left(P_{i}\right)$. Conditions (4.34) are tailored so that $\omega_{\alpha \gamma}$ are all nonnegative, and hence $\hat{\Lambda}(P) u(P) / \hat{\lambda}(P)$ is less than a weighted subaverage (the sum of the averaging coefficients being less than 1 when $q(P)>0)$ of $\hat{\Gamma} u$ at $P$ and at neighboring points. Therefore, if max $\hat{\Gamma} u$ is positive, $\hat{\Lambda}(P) u(P) / \hat{\lambda}(P)$ must be less than this maximum. Furthermore, by (4.33a),

$$
u(P)=\left[\hat{\Lambda}+\sum \lambda_{i} T_{i}+\frac{\mu}{2}\left(T_{10}+T_{01}+T_{-10}+T_{0-1}\right)\right] u(P),
$$


which shows that $u(P)$ is a weighted average of $\hat{\Lambda}(P) u(P) / \hat{\lambda}(P)$ and values of $u$ at neighboring points, and therefore $u(P)$ must also be less than max $\hat{\Gamma} u$. Thus $\hat{\Gamma} u(P)<$ $\max \hat{\Gamma} u$. Q.E.D.

Remark 4.9. Conditions (4.34) could be somewhat relaxed. All we actually need is nonnegativity of the weights $\omega_{\alpha \gamma}$ in the proof, and (4.34) is only one simple way of guaranteeing that. In particular, one would like to change the analysis in the vicinity of boundaries. For instance, if

$$
\alpha L_{h}(P)=\Delta_{h}+r \partial_{x}+s \partial_{y}, \quad|r h|<1,|s h|<1, \alpha>0
$$

and if $L_{h} u(P)>0$, then it can easily be seen that $\Lambda(P)=5^{1 / 2} T_{00}, \mu(P)=1$, and hence $\hat{\Lambda}(P) u(P) / \hat{\lambda}(P)$ and $u(P)$ are both less than weighted averages of $u\left(T_{10} P\right), u\left(T_{01} P\right)$, $u\left(T_{-10} P\right)$ and $u\left(T_{0-1}\right)$. Using this remark for $P$ near boundaries, we immediately deduce the following global maximum principle.

Proposition 4.11. Let $R$ be any bounded domain. Let $L_{h}$ be of the form (4.32) satisfying $q \geqq 0$ and (4.34) in $R_{h, 2}$, of the form (4.35) in $R_{h, 1} / R_{h, 2}$ and of positive type in $R_{h} / R_{h, 1}$. Assume $L_{h} u(P)>0$ for all $P \in R_{h}$, and $u\left(P_{0}\right) \geqq 0$ for some $P_{0} \in B_{h}$. Then the maximum of $u$ must be attained on the boundary $B_{h}$.

Proof. For $Q \in R_{h, 1}$ we define $\hat{\Gamma} u(Q)$ by (4.33). For $Q \in B_{h, 1}$ we set $\hat{\Gamma} u(Q)=u(Q)$. By Proposition 4.10, $\hat{\Gamma} u$ cannot attain its maximum at a point $P \in R_{h, 2}$. By Remark 4.9, the maximum cannot be achieved in $R_{h, 1} / R_{h, 2} . \max \hat{\Gamma} u$ is clearly unattainable in $R_{h} / R_{h, 1}$, where $\hat{\mathrm{\Gamma}} u \equiv u$ and $L_{h}$ is of positive type. Thus, max $\hat{\Gamma} u$ must be attained at some $P_{1} \in B_{h}$. Hence, for all $P \in R_{h}, u(P) \leqq \hat{\Gamma} u(P)<\hat{\Gamma} u\left(P_{1}\right)=u\left(P_{1}\right)$. Q.E.D.

Restrictions (4.34) are mild. If the approximated operator $L$ (cf. (4.0)) is uniformly elliptic with uniformly continuous coefficients, and if $L_{h}$ is constructed in some consistent way (i.e., it is consistent with $L$ and the ratio $b^{+} / b^{-}$is fixed throughout the region, or changes continuously), then (4.34) is automatically satisfied for sufficiently small $h$. Moreover, the coefficients of $L$ need not be even continuous. If $L$ is only uniformly elliptic with bounded coefficients, and if the coefficient of $L_{h}$ (with fixed $b^{+} / b^{-}$) are defined as integral averages of the coefficient of $L$ over a fixed number of mesh cells, then again (4.34) is automatically satisfied for sufficiently small $h$. The smallness of $h$, as well as the fixed number of mesh cells over which each of the integrations is to be performed, depend only on the uniform-ellipticity constant and the bound on the coefficients.

The other conditions required in Proposition 4.11 can also be met in simple ways. For instance, one can replace the original equation in $R_{h} / R_{h, 2}$ by the usual (fivepoint) Laplacian. Then one has also to change the definition of $L_{h}$ in $R_{h, 2} / R_{h, k}$ so that the transition from $L_{h}$ to the Laplacian will be gradual, satisfying (4.34a). The number $k$ here is finite, depending only on the constant of uniform-ellipticity. Therefore, as pointed out by Bramble and Hubbard [4], despite the $O(1)$ local truncation error in $R_{h} / R_{h, k}$, the overall accuracy is still $O\left(h^{2}\right)$. We thus have a way of defining $O\left(h^{2}\right)$ nine-point difference approximations $L_{h}$, to any two-dimensional second-order uniformly-elliptic differential operator $L$, such that the global principle is preserved. This is an improvement over Bramble and Hubbard [4], since they had to use approximations which are not nine-point "box" formulas, containing in fact net-points at distances up to $m h$ apart ( $m$ is finite, depending on the uniform-ellipticity constant), and such approximations are clearly inconvenient from the computational point of view. 
In fact, as in Section 4.2 , it is not necessary to modify $L_{h}$ near the boundary. Although the global maximum principle would not necessarily hold, one can still derive essentially the same a priori estimates. The entire theory for "essentially positive-type" Dirichlet problems [6] can be generalized to operators satisfying (4.34).

4.5. Deeper Decomposition. So far, we have confined our discussion to elliptic difference operators whose principal part is of the form (4.1), where the restriction $b^{+} b^{-} \geqq 0$ has been imposed. This restriction is quite natural; it requires both contributions to the approximation of $b \partial^{2} / \partial x \partial y$ to have the same sign as $b$. Moreover, it can be easily shown that if $b^{+}-b^{-}$were taken too large, namely if $b^{+}-$ $b^{-} \geqq(a+c) / 2$, then $L_{h}$ would not be elliptic in the sense of Thomée [19] and therefore would not possibly have a generalized maximum principle. It is interesting, however, to see what happens to the maximum principle in the opposite case, i.e., when $b^{-}-b^{+}$becomes large (and the condition $b^{+} b^{-} \geqq 0$ is thus violated). In this case, $L_{h}$ is still second-order elliptic, and should have a generalized maximum principle.

Indeed, if $b^{-}-b^{+}>0$, and no matter how big it is, the decomposition (4.2) still holds, with $\lambda_{i}$ as given in (4.4), except that, when $b^{+} b^{-}<0, \lambda_{2}$ turns out negative, since $\lambda_{23}$ and $\lambda_{21}$ in $(4.4 \mathrm{a}, \mathrm{b})$ are both negative. We can still take $\lambda_{0}, \lambda_{1}$ and $\lambda_{3}$ as nonnegative. Furthermore, since $a>0$, it follows from (4.4a) and (4.3) that $\lambda_{01}>\frac{1}{2} b^{-}=$ $\lambda_{31}$ and hence $\lambda_{0}>\lambda_{3}$. Similarly, $\lambda_{0}>\lambda_{1}$. The case considered is that of $b>0$, therefore (4.4e) gives $\lambda_{1} \lambda_{3}>-\lambda_{0} \lambda_{2}$. Also, by (4.4d, f), we have $\lambda_{1}>-\lambda_{2}, \lambda_{3}>-\lambda_{2}$. From (4.4g) we see that when $\lambda_{0}=\lambda_{1}+\lambda_{2}+\lambda_{3}$ (i.e., $\lambda=0$ ) then $a c-b^{2}=0$, while from (4.4d, e, f) it follows that when $\lambda_{0}$ (and hence also $\lambda$ ) decreases, with $\lambda_{1}$, $\lambda_{2}, \lambda_{3}$ remaining fixed, then $a$ and $c$ decreases and $b$ increases, so that $a c-b^{2}$ diminishes. Thus, for $\lambda<0$ we would necessarily have $a c-b^{2}<0$, i.e., ellipticity entails $\lambda>0$. where

Thus, writing $\bar{\lambda}_{2}=-\lambda_{2}$, we have here the decomposition $h^{2} L_{h}=\lambda^{2}-\Lambda^{*} \Lambda$,

$$
\begin{gathered}
\Lambda=\lambda_{0} T_{00}-\lambda_{1} T_{10}+\bar{\lambda}_{2} T_{11}-\lambda_{3} T_{01}, \quad \Lambda^{*}=\lambda_{0} T_{00}-\lambda_{1} T_{-10}+\bar{\lambda}_{2} T_{-1-1}-\lambda_{3} T_{0-1}, \\
\lambda_{0}>\lambda_{1}>\bar{\lambda}_{2}>0, \quad \lambda_{0}>\lambda_{3}>\bar{\lambda}_{2}>0, \\
\lambda=\lambda_{0}-\lambda_{1}+\bar{\lambda}_{2}-\lambda_{3}>0, \quad \lambda_{1} \lambda_{3}>\lambda_{0} \bar{\lambda}_{2} .
\end{gathered}
$$

Neither $L_{h}$ nor $\Lambda$ are of positive type. A positive-type decomposition is obtained, however, by further decomposing $\Lambda$. For instance,

$$
\lambda_{0} \Lambda=H V-\mu T_{11}, \quad \lambda_{0} \Lambda^{*}=H^{*} V^{*}-\mu T_{-1-1},
$$

where

$$
\begin{array}{cc}
H=\lambda_{0} T_{00}-\lambda_{1} T_{10}, & H^{*}=\lambda_{0} T_{00}-\lambda_{1} T_{-10}, \\
V=\lambda_{0} T_{00}-\lambda_{3} T_{01}, & V^{*}=\lambda_{0} T_{00}-\lambda_{3} T_{0-1}, \\
\mu=\lambda_{1} \lambda_{3}-\lambda_{0} \bar{\lambda}_{2}>0 .
\end{array}
$$

This now leads directly to the following local maximum principle:

Proposition 4.12. Let 


$$
L_{h}=a \partial_{x x}+b^{-} \partial_{x y}^{-}+b^{+} \partial_{x y}^{+}+c \partial_{y y},
$$

where $b^{-}-b^{+}>0, b^{-}+b^{+}=2 b>0, b^{2}<a c, a>0$. And let

$$
\Gamma u(Q)=\max \left\{u(Q), \frac{H u(Q)}{\lambda_{0}-\lambda_{1}}, \frac{\Lambda u(Q)}{\lambda}, \frac{H^{*} \Lambda u(Q)}{\left(\lambda_{0}-\lambda_{1}\right) \lambda}\right\} .
$$

If $L_{h} u(P)>0$ then $\Gamma u$ cannot attain its maximum at $P$.

Proof. Like Proposition 4.3, this one also results from a positive representation of each member of $\Gamma u(P)$ in terms of such values at $P$ and at neighboring points. Such a representation is the following:

$$
\begin{gathered}
\lambda_{0} u_{00}=H u_{00}+\lambda_{1} u_{10}, \\
\lambda_{0} H u_{00}=\lambda_{0} \Lambda u_{00}+\lambda_{3} H u_{01}+\mu u_{11}, \\
\lambda_{0} \Lambda u_{00}=H^{*} \Lambda u_{00}+\lambda_{1} \Lambda u_{-10}, \\
\lambda_{0} H^{*} \Lambda u_{00}=-\lambda_{0} h^{2} L_{h} u_{00}+\lambda_{3} H^{*} \Lambda u_{0-1}+\mu \Lambda u_{-1-1}+\lambda_{0} \lambda^{2} u_{00},
\end{gathered}
$$

where $u_{i j}=T_{i} u$. Q.E.D.

5. An $O\left(h^{4}\right)$ Difference Approximation in a General Domain. In this section we apply our general technique to an $O\left(h^{4}\right)$ finite-difference approximation to the Dirichlet problem for elliptic equations whose principal part is the Laplacian. We shall establish both generalized-local and global maximum principles, assuming only suitable $O\left(h^{-1}\right)$ boundedness of the lower-order coefficients. The same problem was treated by Bramble and Hubbard [5] and more fully, but with roughly the same method, by Price [18]. Their result is only slightly weaker than ours; they established a global maximum principle (or "monotonicity") only for mesh-lined regions, while for general domains only a priori estimates were derived. The chief advantage of the present approach is its greater simplicity.

Adopting notation similar to that of the former writers, ${ }^{* *}$ we consider the differential operator

$$
L=\frac{\partial^{2}}{\partial x^{2}}+\frac{\partial^{2}}{\partial y^{2}}-r(x, y) \frac{\partial}{\partial x}+s(x, y) \frac{\partial}{\partial y}-q(x, y), \quad q(x, y) \geqq 0,
$$

in a general bounded domain $R$. The set $R_{h}$ of interior grid points (cf. Section 2) is divided into three disjoint sets $R_{h}^{* *}, B_{h}^{* *}$ and $B_{h}^{*}$, where

$$
\begin{aligned}
R_{h}^{*} & =\left\{P: P \in R_{h} ; T_{\theta 0} P, T_{0 \theta} P \in R \text { for all }|\theta|<1\right\}, \\
R_{h}^{* *} & =\left\{P: P, T_{10} P, T_{01} P, T_{-10} P, T_{0-1} P \in R_{h}^{*}\right\}, \\
B_{h}^{*} & =R_{h} / R_{h}^{*} \quad \text { (which is empty if } R \text { is mesh-lined), } \\
B_{h}^{* *} & =R_{h}^{*} / R_{h}^{* *} .
\end{aligned}
$$

The difference approximation to $L$ is defined by***

** They use $C_{h}, C_{h}^{*}, C_{h}^{* *}$ and $R_{h}$ where we use $B_{h}, B_{h}^{*}, B_{h}^{* *}$ and $R_{h}^{* *}$, respectively.

*** Price, unlike Bramble and Hubbard [5] and the present author, divides $B_{h}^{* *}$ into two sets, with different approximations. The higher-order accuracy thus achieved in one direction is quite redundant, since it is cancelled by the lower accuracy in the other direction. 


$$
\begin{array}{ll}
L_{h}(P)=\partial_{x x}^{(4)}+\partial_{y y}^{(4)}-r(P) \partial_{x}^{(4)}+s(P) \partial_{y}^{(4)}-q(P), & \text { for } P \in R_{h}^{* *}, \\
L_{h}(P)=\partial_{x x}+\partial_{y y}-r(P) \partial_{x}+s(P) \partial_{\nu}-q(P), & \text { for } P \in B_{h}^{* *} .
\end{array}
$$

To define $L_{h}$ for $P \in B_{h}^{*}$ we assume (along with the former authors) that at each direction only one of the discrete neighbors of $P$ is on the boundary. More precisely, if $T_{\lambda 0} P \in B_{h}$ for some $0<|\lambda|<1$, then it is assumed that both $T_{\epsilon 0} P \in R_{h}$ and $T_{2 \epsilon, 0} P \in R_{h}$, where $\epsilon=-\lambda /|\lambda|$. And similarly in the $y$ direction. (This assumption is automatically satisfied if $h$ is small compared to the radius of curvature of $C$. But certain regions with acute corners are thus excluded from consideration.) If, for example, $T_{-\lambda 0} P \in B_{h}$ for some $0<\lambda<1$, we define

$\partial_{x x}^{*}(P)=\frac{1}{h^{2}}\left[-\frac{1-\lambda}{2+\lambda} T_{20}+\frac{2(2-\lambda)}{1+\lambda} T_{10}-\frac{3-\lambda}{\lambda} T_{00}+\frac{6}{\lambda(1+\lambda)(2+\lambda)} T_{-\lambda 0}\right]$,

and

$$
\partial_{x}^{*}(P)=\frac{1}{h}\left[\frac{\lambda}{1+\lambda} T_{10}+\frac{1-\lambda}{\lambda} T_{00}-\frac{1}{\lambda(1+\lambda)} T_{-\lambda 0}\right],
$$

which are $O\left(h^{2}\right)$ approximations to $\partial^{2} / \partial x^{2}$ and $\partial / \partial x$, respectively. Similar definitions are made for the case $T_{\lambda_{0}} P \in B_{h}$. If $T_{\theta 0} P \in R$ for all $|\theta|<1$ we put $\partial_{x x}^{*}(P)=\partial_{x x}$ and $\partial_{x}^{*}(P)=\partial_{x}$. Operators $\partial_{y \nu}^{*}$ and $\partial_{\nu}^{*}$ are similarly defined. Then

$$
L_{h}(P)=\partial_{x x}^{*}(P)+\partial_{y \nu}^{*}(P)-r(P) \partial_{x}^{*}(P)+s(P) \partial_{\nu}^{*}(P)-q(P), \quad \text { for } P \in B_{h}^{*} .
$$

We can now state the global maximum principle. In its proof, a generalized local maximum principle is actually introduced and employed.

Proposition 5.1. If $h$ is small enough, namely

$$
h<\min _{P \in R_{A}}\left\{\frac{2}{3}|r(P)|^{-1}, \frac{2}{3}|s(P)|^{-1},\left[\frac{12}{7} q(P)\right]^{-1 / 2}\right\},
$$

and if

$$
L_{h} u(P) \geqq 0 \quad\left(P \in R_{h}\right),
$$

then the maximum of $u$, if positive, is attained on the boundary $B_{h}$.

Proof. Let $T_{1}=T_{10}, T_{2}=T_{01}, T_{3}=T_{-10}, T_{4}=T_{0-1}$. Denote $P_{i}=T_{i} P$. Introduce auxiliary functions

$$
\begin{aligned}
& v_{i}(P)=\frac{8}{7} u(P)-\frac{1}{7} u\left(P_{i}\right), \quad \text { for } P \in R_{h}^{*}, \\
& w_{i}(P)=\frac{7}{6} u(P)-\frac{1}{6} u\left(P_{i}\right), \quad \text { for } P \in B_{h}^{* *} \text {, } \\
& \bar{u}(P)=\max _{1 \leqq i \leqq 4}\left[u(P), v_{i}(P)\right], \quad \text { if } P \in R_{h}^{* *}, \\
& =\max _{1 \leqq i \leqq 4}\left[u(P), v_{i}(P), w_{i}(P)\right], \text { if } P \in B_{h}^{* *}, \\
& =u(P), \quad \text { if } P \in B_{h}^{*} \cup B_{h} \text {. }
\end{aligned}
$$

We shall show that $\bar{u}$ cannot attain a local positive maximum in $R_{h}$ (unless $u \equiv$ constant).

Indeed, for $P \in R_{h}^{* *}$, inequality (5.3) can be rewritten in the form 


$$
\begin{aligned}
\frac{57+12 q h^{2}}{8} v_{1}(P) \leqq & (1-r h) v_{1}\left(P_{1}\right)+\frac{7-12 q h^{2}}{56} u\left(P_{1}\right)+(1+s h) v_{2}\left(P_{2}\right)+v_{4}\left(P_{2}\right) \\
& +(1+r h) v_{3}\left(P_{3}\right)+v_{1}\left(P_{3}\right)+(1-s h) v_{4}\left(P_{4}\right)+v_{2}\left(P_{4}\right)
\end{aligned}
$$

which shows that $v_{1}(P) \leqq$ a weighted subaverage of $v_{i}\left(P_{i}\right)$ 's and $u\left(P_{1}\right)$. Therefore if $v_{1}(P)=\max \bar{u} \geqq 0$ (and similarly if $v_{k}(P)=\max \bar{u}, k=2,3$ or 4 ), we must also have $\bar{u}\left(P_{i}\right)=\max \bar{u}(i=1,2,3,4)$. Moreover, if

$$
u(P)=\frac{7}{8} v_{1}(P)+\frac{1}{8} u\left(P_{1}\right)=\max \bar{u} \geqq 0
$$

then, clearly, $v_{1}(P)=u\left(P_{1}\right)=\max \bar{u}$, and the above conclusion again follows. Thus, if $\bar{u}(P)=\max \bar{u}$ we must have also $\bar{u}\left(P_{i}\right)=\max \bar{u}(i=1,2,3,4)$.

For $P \in B_{h}^{* *}$ the same conclusion is obtained through the same argument by rewriting (5.3) in the form

$$
\begin{aligned}
\frac{48+12 q h^{2}}{7} w_{1}(P) \leqq & \frac{6-7 r h-2 q h^{2}}{7} u\left(P_{1}\right) \\
& +(2+s h) u\left(P_{2}\right)+(2+r h) u\left(P_{3}\right)+(2-s h) u\left(P_{i}\right),
\end{aligned}
$$

and noting that

$$
u(P)=\frac{6}{7} w_{1}(P)+\frac{1}{7} u\left(P_{1}\right), \quad v_{i}(P)=\frac{48}{49} w_{i}(P)+\frac{1}{49} u\left(P_{i}\right) .
$$

Finally, $\max \bar{u}$ is unattainable also at $P \in B_{h}^{*}$, since there again (5.3) can be rearranged into a positive type inequality, namely

$$
\begin{aligned}
& {\left[\frac{3-\lambda}{\lambda}+\frac{3-\mu}{\mu}+\frac{(1-\lambda) r h}{\lambda}+\frac{(1-\mu) s h}{\mu}+q h^{2}\right] u(P)} \\
& \leqq \\
& \frac{6(1-\lambda)}{2+\lambda} w_{1}\left(P_{1}\right)+\frac{6(1-\mu)}{2+\mu} w_{4}\left(P_{4}\right) \\
& \quad+\left[\frac{2(2-\lambda)-\lambda r h}{1+\lambda}-\frac{7(1-\lambda)}{2+\lambda}\right] u\left(P_{1}\right)+\left[\frac{2(2-\mu)-\mu s h}{1+\mu}-\frac{7(1-\mu)}{2+\mu}\right] u\left(P_{4}\right) \\
& \quad+\frac{6+(2+\lambda) r h}{\lambda(1+\lambda)(2+\lambda)} u\left(T_{-\lambda, 0} P\right)+\frac{6+(2+\mu) s h}{\mu(1+\mu)(2+\mu)} u\left(T_{0, \mu} P\right),
\end{aligned}
$$

where we assumed (with no loss of generality) that both $T_{-\lambda 0} P \in B_{h}$ and $T_{0 \mu} P \in B_{h}$, for some $0<\lambda, \mu \leqq 1$.

Therefore, if $\max \bar{u} \geqq 0$, it must be attained at a point $\bar{P} \in B_{h}$. Hence, for any $P \in R_{h}, u(P) \leqq \bar{u}(P) \leqq \bar{u}(\bar{P})=u(\bar{P})$. Q.E.D.

Remark. Restriction (5.2), which is used in guaranteeing that the inequalities in the proof are of positive-type (i.e., having positive coefficients), could be considerably relaxed, by a more careful, and cumbersome, definition of the auxiliary functions.

6. Simple Illustrations for Parabolic Equations. Generalized local maximum principles for several examples of parabolic difference equations will be exhibited in this section. From these principles, a priori estimates, and hence also stability of the difference schemes, are derivable. The remarkable feature of the method, besides its 
simplicity, is its direct applicability to equations with variable (even discontinuous, in both time and space) coefficients.

6.1. Du Fort-Frankel Approximation to Problems in Two Independent Variables. Consider the general parabolic differential operator in one space dimension

$$
L=-\frac{\partial}{\partial t}+a(x, t) \frac{\partial^{2}}{\partial x^{2}}+b(x, t) \frac{\partial}{\partial x}-c(x, t) \quad(a>0, c \geqq 0) .
$$

Du Fort and Frankel [13] suggest to approximate $L$ by the difference operator

$$
\begin{aligned}
L_{h}= & -\left(\frac{1}{2 k}+\frac{a}{h^{2}}+\frac{c}{2}\right) T_{0}^{1}+\left(\frac{a}{h^{2}}+\frac{b}{2 h}\right) T_{1}^{0} \\
& +\left(\frac{a}{h^{2}}-\frac{b}{2 h}\right) T_{-1}^{0}+\left(\frac{1}{2 k}-\frac{a}{h^{2}}-\frac{c}{2}\right) T_{0}^{-1}
\end{aligned}
$$

where $T_{i}^{i} u(x, t)=u(x+i h, t+j k) . L_{h}$ approximates $L$ (in the sense that for any smooth function $\left.\phi, L_{h} \phi-L \phi \rightarrow 0\right)$ if, and only if, $k=o(h)$. It is assumed that initial conditions are supplied (for example, by using other methods) both for $t=0$ and for $t=k$.

Several generalized local maximum principles can be formulated for $L_{h}$. Perhaps the simplest (but not the best) one is the following:

Proposition 6.1. Let the coefficients in (6.1)-(6.2) satisfy

$$
\begin{gathered}
(2 a-b h)(2 \tilde{a}+\tilde{b} h) \geqq\left(2 a+c h^{2}-h^{2} / k\right)\left(2 \tilde{a}+\tilde{c} h^{2}+h^{2} / k\right), \\
b h<2 a, \quad b<h / k, \quad \tilde{b}<h / k,
\end{gathered}
$$

where $a=a(x, t), b=b(x, t), c=c(x, t), \tilde{a}=a(x-h, t-k), \tilde{b}=b(x-h, t-k)$, $\tilde{c}=c(x-h, t-k)$. Let auxiliary operators be defined by

$$
\Lambda=\left(h^{2} / k+2 a+c h^{2}\right) T_{0}^{1}-(2 a+b h) T_{1}^{0}, \quad \lambda=h^{2} / k+c h^{2}-b h,
$$

If $L_{h} u(x, t)>0$, then $\Gamma u$ cannot attain a positive maximum at $(x, t)$.

Proof. The given inequality $L_{h} u(x, y)>0$ can be rearranged to the form

$$
\Lambda u(x, t)<\frac{2 a-b h}{2 \tilde{a}+\tilde{c} h^{2}+h^{2} / k} \Lambda u(x-h, t-k)+\omega u(x, t-k),
$$

where $\omega>0$ by (6.3a), and $2 a-b h \geqq 0$ by (6.3b). Thus $\Lambda u(x, t) / \lambda u(x, t)$, which is less than a weighted subaverage of $\Lambda u(x-h, t-k)$ and $u(x, t-k)$, cannot attain $\max \Gamma u \geqq 0$. Furthermore, $u(x, t+k)$ is a weighted average of $\Lambda u(x, t) / \lambda(x, t)$ and $u(x+h, t)$, and therefore it also cannot attain $\max \Gamma u \geqq 0$. Q.E.D.

Remarks. Restrictions (6.3) can be relaxed by using more sophisticated $\Gamma$. Even so, these restrictions are fairly mild. For example, if $a, b, c, a_{t}+a_{x}$ and $b_{t}+b_{x}$ are all bounded, then for $h$ and $k=o(h)$ sufficiently small (6.3) is automatically satisfied, since $a-\tilde{a}=O(h)$ and $a \tilde{b}-\tilde{a} b=O(h)$.

The stability of $L_{h}$ is an easy consequence of the above proposition. It is contained in the following a priori estimate for the Cauchy problem, from which convergence estimates are also deducible. 
Proposition 6.2. Let $L_{h}$ be defined by (6.2) and satisfy (6.3). Assume $\bar{u}$ is a constant such that

$$
\begin{aligned}
& u(x, 0) \leqq \bar{u} \\
& \frac{(2 a-b h) u(x-h, k)-\left(2 a+c h^{2}-h^{2} / k\right) u(x, 0)}{h^{2} / k-b h-c h^{2}} \leqq \bar{u} \quad(-\infty<x<\infty),
\end{aligned}
$$

where $a=a(x, k), b=b(x, k), c=c(x, k)$. Assume also

$$
L_{h} u(x, t) \geqq-\bar{l} \quad(-\infty<x<\infty, k<t<i),
$$

where $x / h, t / k$ and $t / k$ are all integers. Then

$$
u(x, t) \leqq t \bar{l}+\bar{u} \quad(-\infty<x<\infty, 0 \leqq t \leqq \bar{t}) .
$$

Proof. It is easy to check that $L_{h} t=-1-c t \leqq-1$, and hence $L_{h} \phi>0$, where $\phi=u-t \bar{l}$. For any fixed $t, u(x, t)$ is a bounded function of $x$, since $L_{h}$ is an explicit difference operator. Therefore

$$
m_{t}=\sup _{x} \phi(x, t) \quad \text { and } \quad \Gamma_{t}=\sup _{x} \Gamma \phi(x, t)
$$

are finite. Clearly, $m_{t} \leqq \Gamma_{t-k}$. For any fixed $t>k$ and preassigned $\epsilon$, there exists a point $(x, t)$ such that $\Gamma u(x, t)>\Gamma_{t}-\epsilon$. Applying Proposition 6.1 for the function $\phi$ at that point $(x, t)$ and then letting $\epsilon \rightarrow 0$, we get that $\Gamma_{t}$ is either negative or less than $\Gamma_{t-k}$ or less than $m_{t-k} \leqq \Gamma_{t-2 k}$. From (6.7), we have $m_{0} \leqq \bar{u}$ and also $m_{k}<\bar{u}$. From (6.2) and (6.7), we can see that $\Gamma_{k}$ is less than $\bar{u}$. Thus, $\phi(x, t) \leqq m_{t} \leqq \Gamma_{t-k} \leqq \bar{u}$ for all $k<t \leqq i$. Q.E.D.

6.2. Implicit Approximations to Problems in Three Independent Variables. The general second-order parabolic operator in two space variables is

$L=-\frac{\partial}{\partial t}+a \frac{\partial^{2}}{\partial x^{2}}+2 b \frac{\partial^{2}}{\partial x \partial y}+c \frac{\partial^{2}}{\partial y^{2}}+r \frac{\partial}{\partial x}+s \frac{\partial}{\partial y}-q \quad\left(b^{2}<a c, a>0\right)$,

where the coefficients $a, b, c, r, s, q$ are functions of $x, y$ and $t$. Consider the implicit finite-difference approximations of the form

$$
\begin{aligned}
L_{h}=-\partial_{t}^{-}+a \partial_{x x}+b^{+} \partial_{x y}^{+}+b^{-} \partial_{x y}^{-}+c \partial_{y y}+r \partial_{x}+s \partial_{y}-q \\
\left(b^{+}+b^{-}=2 b, b^{+} b^{-} \geqq 0\right)
\end{aligned}
$$

where

$$
\partial_{\imath}^{-} \phi(x, y, t)=[\phi(x, y, t)-\phi(x, y, t-k)] / k
$$

and

$$
\partial_{x} \phi(x, y, t)=[\phi(x+h, y, t)-\phi(x-h, y, t)] / 2 h,
$$

with similar definitions for all other spatial difference quotients (cf. Section 2). We shall see that essentially the same maximum principles hold for (6.10) as for the corresponding elliptic operator.

First it is clear that, as in Proposition 4.9, (6.10) can be decomposed in the form

$$
L_{h}(Q)=-\partial_{t}^{-}+\frac{\lambda^{2}-\Lambda^{*} \Lambda}{h^{2}}+\mu \Delta_{h}+r \partial_{x}+s \partial_{\nu}-q, \quad \mu=\lambda^{2} / 5>0,
$$


where (cf. (4.32); here, because of the additional term $-\partial_{t}^{-}$, we cannot make the normalization $\lambda_{0}=1$ )

$$
\begin{gathered}
\Lambda=\lambda_{0} T_{00}-\lambda_{1} T_{10}-\lambda_{2} T_{11}-\lambda_{3} T_{01}-\lambda_{4} T_{-11}-\lambda_{5} T_{-10}, \\
\Lambda^{*}=\lambda_{0} T_{00}-\lambda_{1} T_{-10}-\lambda_{2} T_{-1-1}-\lambda_{3} T_{0-1}-\lambda_{4} T_{1-1}-\lambda_{5} T_{10}, \\
\lambda=\lambda_{0}-\lambda_{1}-\lambda_{2}-\lambda_{3}-\lambda_{4}-\lambda_{5}>0, \quad \lambda_{i}=\lambda_{i}(Q) \geqq 0, \\
T_{i j} \phi(x, y, t)=\phi(x+i h, y+j h, t),
\end{gathered}
$$

and where, for any net point $Q$, either $\lambda_{1}(Q)=\lambda_{2}(Q)=0$ or $\lambda_{4}(Q)=\lambda_{5}(Q)=0$. We also introduce the auxiliary operators $\hat{\Lambda}$ and $\hat{\Gamma}$,

$$
\begin{gathered}
\hat{\Lambda}(Q)=\Lambda(Q)-\frac{1}{2} \mu(Q)\left(T_{10}+T_{01}+T_{-10}+T_{0-1}\right), \\
\hat{\lambda}(Q)=\lambda(Q)-2 \mu(Q), \\
\hat{\Gamma} u(Q)=\max \{u(Q), \hat{\Lambda}(Q) u(Q) / \hat{\lambda}(Q)\},
\end{gathered}
$$

in terms of which we can state the following local maximum principle.

Proposition 6.3. Let $L_{k}$, of the form (6.11)-(6.12), be such that

$$
\begin{array}{ccc}
\text { (6.14a) } & \left|\lambda_{i} / \lambda_{0}-T_{\alpha \gamma}\left(\lambda_{i} / \lambda_{0}\right)\right| \leqq \mu / 3 \lambda_{0}^{2} & (-1 \leqq \alpha, \gamma \leqq 1,1 \leqq i \leqq 5), \\
\text { (6.14b) } & |3 h r| \leqq \mu, \quad|3 h s| \leqq \mu, & \frac{3}{2} h^{2}(q+1 / k) \leqq \mu .
\end{array}
$$

If $L_{h} u(P)>0$ and $q(P) \geqq 0$, then $\hat{\Gamma} u($ defined by (6.13)) cannot attain a positive maximum at $P$.

Proof. Dividing the equation through by $\lambda_{0}^{2}$, the situation here is the same as in Proposition 4.10, with $\lambda, \lambda_{i}, \mu, r, s$ and $q$ of that proposition replaced here by $\lambda / \lambda_{0}$, $\lambda_{i} / \lambda_{0}, \mu / \lambda_{0}^{2}, r / \lambda_{0}^{2}, s / \lambda_{0}^{2}$ and $(q+1 / k) / \lambda_{0}^{2}$, respectively, and with the additional term $u(x, y, t-k) / \lambda_{0}^{2} k$. Since this term clearly has a positive weight, the same conclusions follow, namely that both $u$ and $\hat{\Lambda} u / \hat{\lambda}$ at $P$ are less than weighted subaverages of their values at neighboring points, and therefore $\hat{\Gamma} u(P)<\max \hat{\Gamma} u$. Q.E.D.

Conditions (6.14) are mild. They are automatically satisfied for sufficiently small $h$ ( $k$ need not be small!) provided $L$ is uniformly parabolic and the coefficients of $L_{h}$ (with continuous ratio $b^{+} / b^{-}$) are integral averages of the coefficients of $L$ over a certain number of mesh cells. This number, and the smallness of $h$, depend only on the uniform parabolicity constant.

From Proposition 6.3 one can deduce global estimates, and sometimes even global maximum principles, e.g., similar to Proposition 4.11. Using local maximum principles one can also easily derive estimates for difference quotients, as in Brandt [9].

Local maximum principles obviously imply stability and convergence of the associated discrete schemes. It is still an open problem whether the converse is generally true, namely, whether a generalized-local maximum principle can be formulated for any stable second-order parabolic difference equation.

Department of Applied Mathematics

Weizmann Institute of Science

Rehovot, Israel

1. J. H. Bramble, "Fourth order finite difference analogues of the Dirichlet problem for Poisson's equation in three and four dimensions," Math. Comp., v. 17, 1963, pp. 217-222. MR 28 \#3551. 
2. J. H. BRAmble, "On the convergence of difference approximations for second order uniformly elliptic operators," SIAM-AMS Proc., vol. II, Amer. Math. Soc., Providence, R.I., 1970, pp. 201-209. MR 41 \#4828.

3. J. H. Bramble \& B. E. Hubbard, "On the formulation of finite difference analogues of the Dirichlet problem for Poisson's equation," Numer. Math., v. 4, 1962, pp. 313-327. MR 26 \#7157.

4. J. H. BRAmble \& B. E. Hubbard, "A theorem on error estimation for finite difference analogues of the Dirichlet problem for elliptic equations," Contributions to Differential Equations, v. 2, 1963, pp. 319-340. MR 27 \#2114.

5. J. H. Bramble \& B. E. Hubbard, "New monotone type approximations for elliptic problems," Math. Comp., v. 18, 1964, pp. 349-367. MR 29 \#2982.

6. J. H. Bramble, B. E. Hubbard \& V. Thomée, "Convergence estimates for essentially positive type discrete Dirichlet problems," Math. Comp., v. 23, 1969, pp. 695-709. MR 42 \#1350.

7. A. Brandt, "Estimates for difference quotients of solutions of Poisson type difference equations," Math. Comp., v. 20, 1966, pp. 473-499. MR 34 \#4732.

8. A. BRANDT, "Interior estimates for second-order e"!:ptic differential (or finite-difference) equations via the maximum principle," Israel J. Math., J. 7, 1969, pp. 95-121. MR 40 \#6052.

9. A. BRANDT, "Interior Schauder estimates for parabolic differential (or difference-) equations via the maximum principle," Israel J. Math., v. 7, 1969, pp. 254-262. MR 40 \#3044.

10. P. G. Ciarlet, "Discrete maximum principle for finite-difference operators," Aequationes Math., v. 4, 1970, pp. 338-352. MR 45 \#1404.

11. L. Collatz, "Bemerkungen zur Fehlerabschätzung für das Differenzenverfahren bei partiellen Differentialgleichungen," Z. Angew. Math. Mech., v. 13, 1933, pp. 56-57.

12. R. Courant \& D. Hilbert, Methods of Mathematical Physics. Vol. II. Partial Differential Equations, Interscience, New York, 1962. MR 25 \#4216.

13. E. C. DU FCRT \& S. P. Frankel, "Stability condition in the numerical treatment of parabolic differential equations," Math. Tables Aids Comput., v. 7, 1953, pp. 135-152. MR 15, 474.

14. G. Forsythe \& W. WAsOW, Finite-Difference Methods for Partial Differential Equations, Wiley, New York, 1960. MR 23 \# B3156.

15. S. GERSCHGORIN, "Fehlerabschätzung für das Differenzenverfahren zur Lösung partieller Differentialgleichungen,” Z. Angew. Math. Mech., v. 10, 1930, pp. 373-382.

16. P. LAASONEN, "On the discretization error of the Dirichlet problem in a plane region with corners," Ann. Acad. Sci. Fenn. Ser. A I, No. 408, 1967, pp. 1-16. MR 38 \# 335.

17. T. MotZkin \& W. WASOW, "On the approximation of linear elliptic differential equations by difference equations with positive coefficients," J. Mathematical Phys., v. 31, 1953, pp. 253-259. MR 14, 693.

18. H. S. PrICE, "Monotone and oscillation matrices applied to finite difference approximations," Math Comp., v. 22, 1968, pp. 489-516. MR 38 \#875.

19. V. Tноме́E, "Elliptic difference operators and Dirichlet problem," Contributions to Differential Equations, v. 3, 1964, pp. 301-324. MR 29 \#46. 